\title{
Unilateral and equitransitive tilings by squares of four sizes
}

\author{
Casey Mann \\ University of Washington - Bothell, School of STEM, \\ 18115 Campus Way N.E., Bothell, WA 98011, USA \\ Joseph DiNatale \\ Armstrong Atlantic State University, USA \\ Emily Peirce \\ Baylor University, USA \\ Ellen Vitercik \\ Columbia University, USA
}

Received 3 April 2014, accepted 15 July 2014, published online 18 August 2015

\begin{abstract}
D. Schattschneider proved that there are exactly eight unilateral and equitransitive tilings of the plane by squares of three distinct sizes. This article extends Schattschneider's methods to determine a classification of all such tilings by squares of four different sizes. It is determined that there are exactly 39 unilateral and equitransitive tilings by squares of four different sizes.
\end{abstract}

Keywords: Tilings, equitransitive, unilateral, squares.

Math. Subj. Class.: 05B45, 52C20, 54E15, 05C15

\section{Introduction}

A two-dimensional tiling, $\mathscr{T}$, is a countable collection of closed topological disks $\left\{T_{i}\right\}$, called tiles, such that the interiors of the $T_{i}$ are pairwise disjoint and the union of the $T_{i}$ is the Euclidean plane. A symmetry of $\mathscr{T}$ is any planar isometry that maps every tile of $\mathscr{T}$ onto a tile of $\mathscr{T}$. Two tiles $T_{1}$ and $T_{2}$ are equivalent if there exists a symmetry of $\mathscr{T}$ that

E-mail addresses: cmann@uwb.edu (Casey Mann),jd4732@stu.armstrong.edu (Joseph DiNatale), EmilyPeirce@baylor.edu (Emily Peirce), emv2126@columbia.edu (Ellen Vitercik) 
maps $T_{1}$ onto $T_{2}$. The collection of all tiles of $\mathscr{T}$ that are equivalent to $T_{1}$ is called the transitivity class of $T_{1}$. $\mathscr{T}$ is equitransitive if each set of mutually congruent tiles forms one transitivity class.

This article will concern only tilings of the plane by squares of a few different sizes. A connected segment formed by the intersection of two squares of $\mathscr{T}$ will be called an edge of $\mathscr{T}$, and the endpoints of the edges are called vertices of $\mathscr{T} . \mathscr{T}$ is unilateral if each edge of the tiling is a side of at most one tile, meaning that if two congruent tiles meet along an edge, they are never incident along the full length of that edge. The acronym UETn will refer to a unilateral and equitransitive tiling by squares of $n$ distinct sizes.

A classification of all UET3 tilings is given in [3]. There are only eight UET3 tilings, shown in Figure 1. Because the classification of UET4 tilings is based on the methology of [3], it will be helpful to outline those methods here. First, some notation and terminology must be introduced.

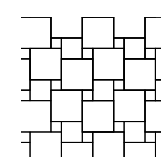

(a) (c.c.c.b, c.a.c.c.a.c, a.c.a.c.b.a.c.b)

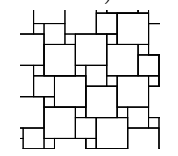

(e) (c.c.c.b, a.c.c.a.c.c, a.b.c.a.b.c.a.c)

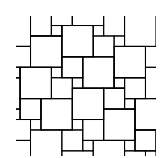

(b) (c.c.b.b, c.a.b.a.c.c, b.c.b.a.c.b.a.c)

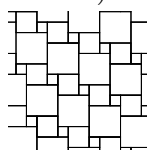

(f) (c.b.c.b, a.c.c.a.c.c, a.b.c.b.a.b.c.b)

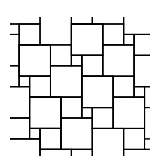

(c) (c.b.c.b, c.c.a.c.c, b.c.b.c.b.a.b.c)

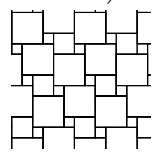

(g) (c.c.c.b, a.c.c.a.c.c, a.c.a.c.a.b.c.b.a)

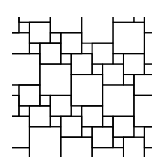

(d) (c.b.b.b, a.c.c.a.b.a.b, a.b.c.b.a.b.c.b)

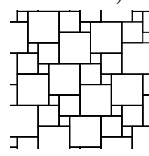

(h) (c.c.b.b,a.c.c.c.a.b, a.b.c.b.c.b.a.c)

Figure 1: The eight UET3 tilings classified in [3].

Let $\mathscr{T}$ be a UET4 tiling of squares with side lengths $a<b<c<d$. The skeleton of $\mathscr{T}$ is the union of all of the edges of the tiling $\mathscr{T}$. A vortex is a tile $T \in \mathcal{T}$ for which each edge of the tile is extendable within the skeleton of $\mathscr{T}$ in exactly one direction, given an orientation of $T$, as in Figure 2.

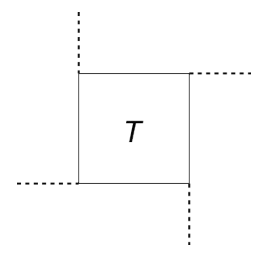

Figure 2: A vortex tile

A corona of a tile $T$ in a tiling $\mathscr{T}$ consists of all tiles in $\mathscr{T}$ whose intersection with $T$ is nonempty. The corona signature of $T$ is an ordered list of the sizes of the tiles in $T$ 's corona. In a UET4 tiling the coronas of any two congruent copies of $T$ must be congruent due to equitransitivity, so the corona signature of $T$ unambiguously describes the corona of any tile in $\mathscr{T}$ that is congruent to $T$. A sample $d$ corona (i.e. a corona of a $d$ tile) and its corresponding corona signature are given in Figure 3. The corona signatures of the eight 
UET3 tilings shown in Figure 1 are given as a triplet ( $a$ corona signature, $b$ corona signature, $c$ corona signature). Cyclic permutations of a signature, as well as cyclic permutations of a signature read in reverse, are considered equivalent to the original signature.

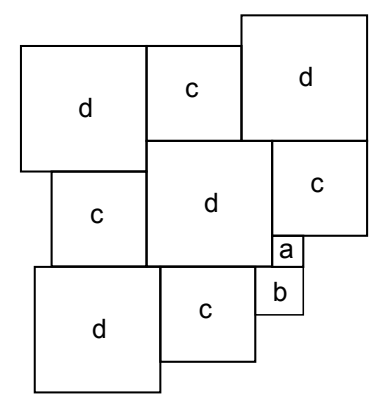

Figure 3: This $d$ corona has signature c.d.c.a.b.c.d.c.d.

\subsection{Schattschneider's Method}

The method of classification used in [3] to find all UET3 tilings can be roughly described as follows:

1. Determine all extendable $a, b$, and $c$ coronas.

2. Determine which 3-tuples of extendable $a, b$, and $c$ coronas are compatible in terms of their corona signatures.

3. Determine which 3-tuples of compatible $a, b$, and $c$ corona signatures give rise to tilings (and how many).

A similar process will be followed in this article (with the addition of finding all extendable $d$ corona signatures). It is to be expected that the scope of the UET4 classification problem is broader in size than the UET3 problem; as a result, the problem is solved through two major cases. These are the cases where:

1. $a$ and $b$ are adjacent.

2. $a$ and $b$ are not adjacent.

Two tiles are adjacent if their intersection is an edge of the tiling. Sections 2 - 4 concern the case when $a$ tiles and $b$ tiles are adjacent. While the case where $a$ tiles and $b$ tiles are not adjacent employs themes established these sections, the differences between these cases are sufficient to require a separate analysis; this is done in Section 5.

\section{UET4 tilings in which $a$ tiles and $b$ tiles are adjacent}

The bulk of the work done in classifying all UET4 tilings is enumerating all possible $a, b, c$, and $d$ coronas. This job is made manageable by first establishing some necessary equations relating $a, b, c$, and $d$. These equations are established in Subsection 2.1. After establishing a finite set of possible equations relating $a, b, c$, and $d$, the coronas corresponding to these equations are found; this is described in Section 3. Finally, once a set of coronas 
corresponding to an equation or equations is established, the process for constructing the possible tilings is described in Section 4.

One fact that will be used throughout the article comes from [2], and can be stated as follows.

Lemma 2.1. Let $\mathscr{T}$ be a UET4 tiling of squares with side lengths $a<b<c<d$. Then all $a$ and $b$ tiles of $\mathscr{T}$ are vortices.

\subsection{Equations relating $a, b, c$, and $d$}

Lemma 2.2. Let $\mathscr{T}$ be a UET4 tiling in which $a$ and $b$ are adjacent. Then $a+b=c$ or $a+b=d$.

Proof. Begin by examining an $a$ corona. Because $a$ and $b$ tiles are adjacent vortices, these two tiles must meet at a corner as shown in Figure 4a. The dashed lines depict the necessary skeletal extension in $\mathscr{T}$ required by the vortex condition on the $a$ and $b$ tiles. It is clear that a tile or group of tiles must fill the length indicated in Figure 4 a exactly in order for these vortex conditions to hold.

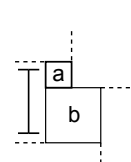

(a)

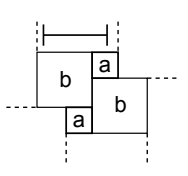

(b)

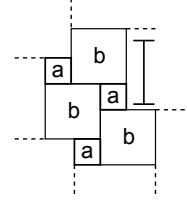

(c)

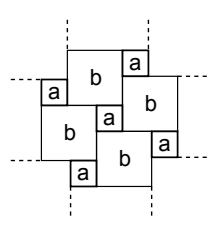

(d)

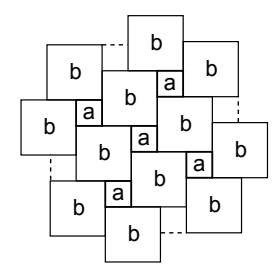

(e)

Figure 4

Suppose $a+b \neq c$ and $a+b \neq d$. Neither a $c$ tile nor a $d$ tile can fill the space indicated exactly, so some combination of $a$ and $b$ tiles must be used instead. In fact, the vortex condition requires that exactly two such tiles be used, and unilaterality implies that exactly one $a$ tile and one $b$ tile must be used. This yields the arrangement shown in Figure 4b. The length indicated in $4 \mathrm{~b}$ brings up the same issue, and following the same logic it is seen that the arrangement in Figure $4 c$ is the only valid arrangement for this space. The same is true for the length indicated in Figure 4c, yielding the full $a$ corona found in Figure 4d. Having now completed an a corona, equitransitivity tells us that every $a$ corona in $\mathscr{T}$ must be identical to this, which generates the patch seen in Figure 4e. The only possible unilateral and equitransitive tiling that this patch admits contains only $a$ and $b$ tiles and is therefore UET2.

This gives rise to two subcases within the case of $a$ and $b$ being adjacent, namely that where $a+b=c$ and that where $a+b=d$. These two cases are considered in turn.

\subsection{1 $a+b=c$}

The following two subcases of that when $a$ and $b$ are adjacent and the equation $a+b=c$ is satisfied are considered separately: 
1. The $d$ tile is not a vortex.

2. The $d$ is a vortex.

\section{The $d$ tile is not a vortex:}

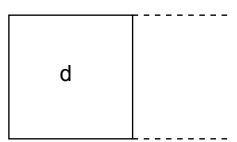

Figure 5: The extension of a non-vortex $d$ tile.

If $d$ is not a vortex, then is must have a pair of parallel edges that extend into the skeleton of $\mathscr{T}$ as in Figure 5. There must be some combination of $a, b$, and $c$ tiles which fit perfectly between the dashed lines in Figure 5. Since $a$ and $b$ are vortices, they must share a corner with the $d$ tile and therefore one of their edges must be contained in a dashed line. There are exactly five possible ways to fill the region, all of which are shown in Figure 6.

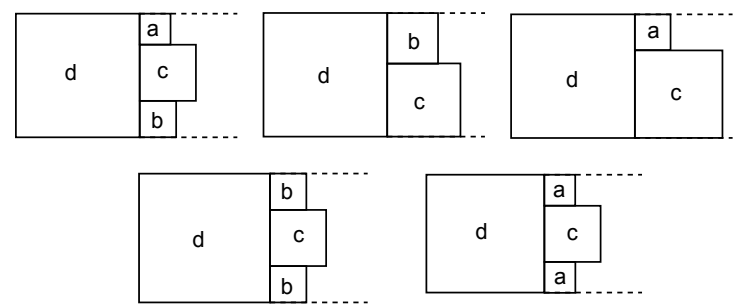

Figure 6: All possible ways of filling the region between the dashed lines.

This then gives us exactly five possible relationships for $d$ if it is not a vortex:

1. $d=a+b+c=2 a+2 b$

2. $d=b+c=a+2 b$

3. $d=a+c=2 a+b$

4. $d=2 b+c=3 b+a$

5. $d=2 a+c=3 a+b$

\section{The tile $d$ is a vortex:}

If $d$ is a vortex, then the $d$ corona must contain either an $a$ or a $b$, as explained below; furthermore, there must be an $a$ or $b$ tile that shares a corner with the $d$ tile to satisfy the pertinent vortex conditions, as depicted in Figure 7.

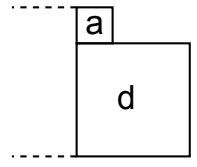

(a)

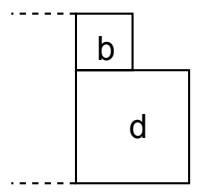

(b)

Figure 7 
If this was not the case, then the tiling would be UET2. To see this, notice that since $d$ is a vortex, there must be tiles which line up exactly with the doted lines in Figure 8a. If there are no $a$ or $b$ tiles in the corona of the $d$ tile, these tiles must be $c$ tiles, as in Figure $8 \mathrm{~b}$. Finally, the rest of the corona must be made up by $d$ tiles, as in Figure 8c. This patch can only be extended to a UET2 tiling. Therefore, there must be at least one $a$ or $b$ tile which shares a corner with the $d$ tile.

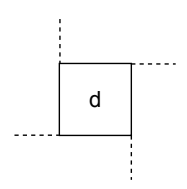

(a)

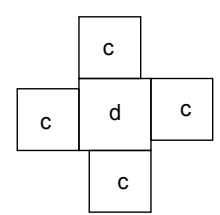

(b)

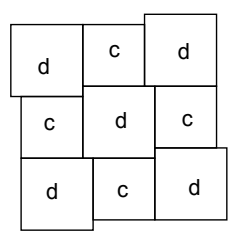

(c)

Figure 8: A UET2 corona.

Since $a, b$, and $d$ are all vortices in this subcase, there must be a combination of tiles that fits perfectly between the dashed edges indicating edge extentions into the skeleton of the tiling in Figure 7. No more than three tiles may fit in this space, for if there were four or more, then the two or more tiles sandwiched in the middle would have to be nonvortices. However, only $c$ can be a non-vortex, and two $c$ tiles cannot meet edge-to-edge by the unilateral condition.

Only specific configurations of tiles can fit between the dashed lines. By examining these configurations and using simple algebra, it is easy to enumerate the possible relationships between $d$ and the smaller square sizes that would allow for a tiling. This analysis is summarized in Table 1.

Of course, it is possible for a d tile to share a corner with a $a$ or $b$ tile even if $d$ is not a vortex, which is why some $d$ relations are repeated from the case where $d$ tiles were not vortices. In this case, however, only those $d$ relationships for which the tile is necessarily a vortex are considered, so any repeated relationships are disregarded, resulting in the following complete list of $d$ relationships when $c=a+b$ :

1. $d=a+b+c=2 a+2 b$

2. $d=b+c=a+2 b$

3. $d=a+c=2 a+b$

4. $d=2 b+c=3 b+a$

5. $d=2 a+c=3 a+b$

6. $d=2 b$

7. $d=3 a$

8. $d=3 b$

\subsubsection{Aside: $a: b$ side length proportions}

When $a+b=c$ and $a$ and $b$ are adjacent, there are certain tile configurations that are possible only when the size of $b$ is specifically related to the size of $a$. By examining 


\begin{tabular}{|c|c|c|c|c|}
\hline $\begin{array}{l}\text { All } \\
\text { possible } \\
\text { combina- } \\
\text { tions of } \\
\text { two or } \\
\text { three tiles }\end{array}$ & $\begin{array}{l}\text { Could they } \\
\text { fit between } \\
\text { the dashed } \\
\text { lines in } \\
\text { Figure } 7 a ?\end{array}$ & $\begin{array}{l}\text { If yes, what } d \\
\text { relations are } \\
\text { required for the } \\
\text { configuration to fit } \\
\text { perfectly? }\end{array}$ & $\begin{array}{l}\text { Could they } \\
\text { fit between } \\
\text { the dashed } \\
\text { lines in } \\
\text { Figure } 7 \mathrm{~b} \text { ? }\end{array}$ & $\begin{array}{l}\text { If yes, what } d \text { re- } \\
\text { lations are required } \\
\text { for the configura- } \\
\text { tion to fit perfectly? }\end{array}$ \\
\hline$a+b$ & No & & No & \\
\hline$a+c$ & No & & No & \\
\hline$a+d$ & Yes & $\begin{array}{l}a+d=a+d \\
\rightarrow \text { No new infor- } \\
\text { mation }\end{array}$ & No & \\
\hline $\begin{array}{l}b+c= \\
2 b+a\end{array}$ & Yes & $\begin{array}{l}a+d=a+2 b \\
\rightarrow d=2 b\end{array}$ & No & \\
\hline$b+d$ & No & & Yes & $\begin{array}{l}b+d=b+d \\
\rightarrow \text { No new infor- } \\
\text { mation }\end{array}$ \\
\hline$c+d$ & No & & No & \\
\hline $\begin{array}{l}a+c+a= \\
3 a+2 b\end{array}$ & No & & Yes & $\begin{array}{l}b+d=3 a+b \\
\rightarrow d=3 a\end{array}$ \\
\hline $\begin{array}{l}a+c+b= \\
2 a+2 b\end{array}$ & Yes & $\begin{array}{l}a+d=2 a+2 b \\
\rightarrow d=a+2 b\end{array}$ & Yes & $\begin{array}{l}b+d=2 a+2 b \\
\rightarrow d=2 a+b\end{array}$ \\
\hline$a+c+d$ & No & & No & \\
\hline $\begin{array}{l}b+c+b= \\
a+3 b\end{array}$ & Yes & $\begin{array}{l}a+d=a+3 b \\
\rightarrow d=3 b\end{array}$ & No & \\
\hline$b+c+d$ & No & & No & \\
\hline
\end{tabular}

Table 1

these specific configurations for each case, certain $a: b$ ratios are determined that must be considered; such ratios are determined when a set of tiles must fit perfectly between the extended edges of two vortices. These specific tile configurations are shown in Figure 9 and Figure 10. Tables showing the arithmetic used to find the $a: b$ ratios are provided as well. In the first row of both tables, the eight $d$ relations previously generated for this case $(a+b=c$ and $a$ and $b$ are adjacent) are considered. In the leftmost column, the configurations as well as the general proportions they necessitate are listed. For example, in Figure 9a, a $b$ tile and a $c$ tile fit perfectly above two $a$ 's and a $c$. For this configuration to occur, $b+c=2 a+c$. Therefore, $b=2 a$.

It should be noted that the subcases within the case where $a+b=c$, each of which is technically given by a different $b$ side length, are not considered within the actual corona construction process as separate cases. Instead, the reader should bear them in mind as corona construction begins within the appropriate specified subcase. It should additionally be noted that these $a: b$ side relations are only pertinent when $a+b=c$. They are not considered in the case where $a+b=d$, which follows.

\subsection{3 $a+b=d$}

Lemma 2.3. If $a+b=d$, then $c$ must be a vortex.

Proof. Suppose the $c$ tile is not a vortex. Then two edges of any $c$ tile must extend into the 


\begin{tabular}{|c|c|c|c|c|c|c|c|c|c|}
\hline 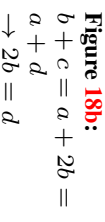 & 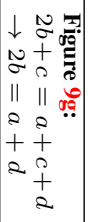 & 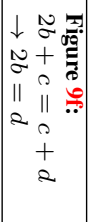 & 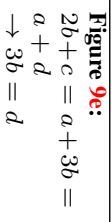 & 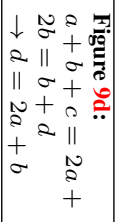 & 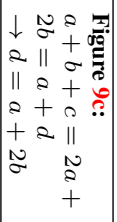 & 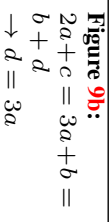 & 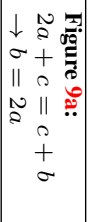 & 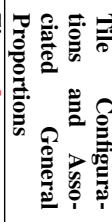 & \\
\hline 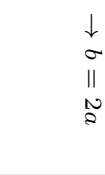 & $\begin{array}{ccc}\downarrow & N \\
\sigma & \| \\
\| & \omega \\
\omega & \omega \\
\sigma & + \\
& \sigma\end{array}$ & 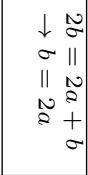 & 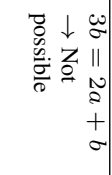 & 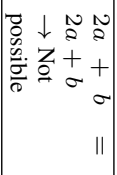 & 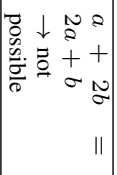 & 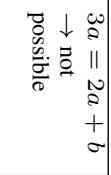 & $\begin{array}{c}\downarrow \\
\sigma \\
11 \\
0 \\
0\end{array}$ & & $\sigma$ \\
\hline 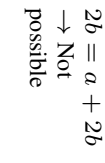 & 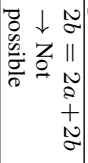 & 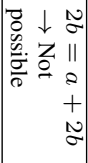 & 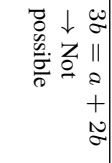 & 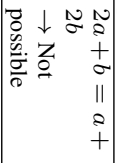 & 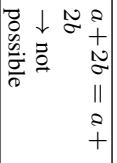 & 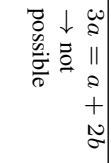 & $\begin{array}{l}\downarrow \\
\sigma \\
11 \\
N \\
2\end{array}$ & & \\
\hline $\begin{array}{l}\downarrow \\
\sigma \\
\| \\
c \\
\&\end{array}$ & 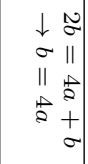 & 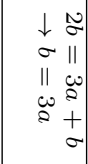 & \begin{tabular}{cc|}
$\downarrow$ & $\omega$ \\
$\sigma$ & $\sigma$ \\
$\sigma$ & $\|$ \\
$\|$ & $\omega$ \\
$N \mid \omega$ & $\sigma$ \\
$\sigma$ & + \\
& $\sigma$
\end{tabular} & 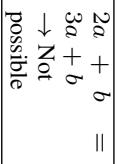 & $\begin{array}{lll}\downarrow & \omega & \sigma \\
\sigma & a \\
\sigma & + \\
\| & \sigma \\
N & \sigma \\
\tilde{N} & & \\
& & \|\end{array} \mid$ & 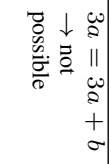 & $\begin{array}{l}\downarrow \\
\sigma \\
11 \\
\tilde{a}\end{array}$ & & 리 \\
\hline 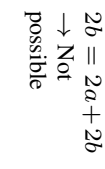 & 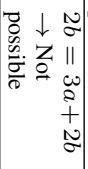 & 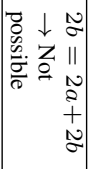 & 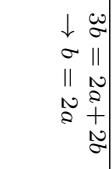 & 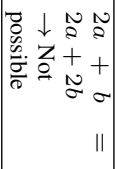 & 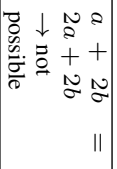 & 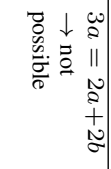 & $\begin{array}{l}\downarrow \\
\sigma \\
11 \\
\text { है }\end{array}$ & & II \\
\hline 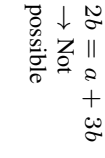 & 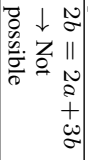 & 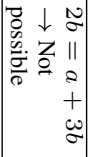 & 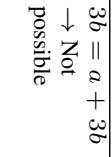 & 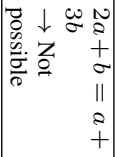 & 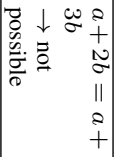 & 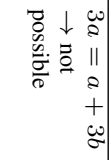 & $\begin{array}{l}\downarrow \\
\sigma \\
11 \\
\text { है }\end{array}$ & & $\begin{array}{l}8 \\
11 \\
8\end{array}$ \\
\hline $\begin{array}{l}\mathcal{N} \\
\| \\
\mathcal{\sigma}\end{array}$ & 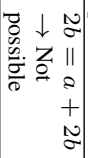 & 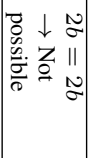 & 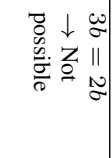 & 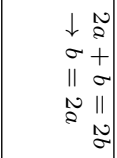 & 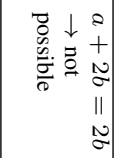 & 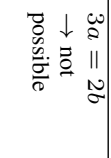 & $\begin{array}{l}\downarrow \\
\sigma \\
11 \\
\tilde{2} \\
2\end{array}$ & & \\
\hline 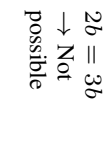 & 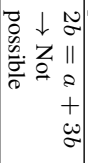 & 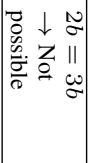 & 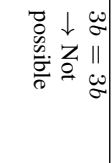 & 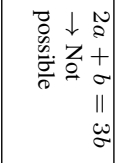 & 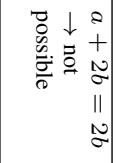 & 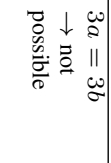 & 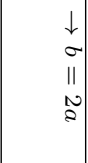 & & \\
\hline $\begin{array}{c}\downarrow \\
\sigma \\
\| \\
N \mid a \\
\alpha\end{array}$ & 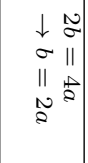 & 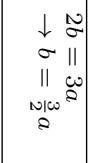 & 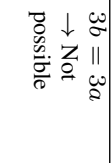 & 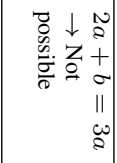 & 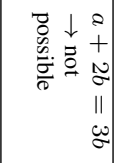 & 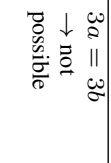 & $\begin{array}{l}\downarrow \\
\sim \\
11 \\
N \\
2\end{array}$ & & 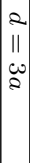 \\
\hline
\end{tabular}




\begin{tabular}{|c|c|c|c|c|c|c|c|}
\hline 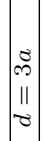 & & 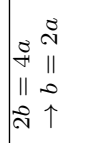 & 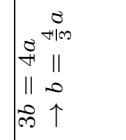 & $\begin{array}{l}\stackrel{2}{N} \\
\| 1 \\
0 \\
\uparrow\end{array}$ & & $\begin{array}{l}\tilde{N} \\
\| \\
11 \\
0 \\
\uparrow\end{array}$ & $\begin{array}{l}\infty \\
\infty \\
11 \\
0 \\
\uparrow\end{array}$ \\
\hline $\begin{array}{l}\stackrel{0}{c} \\
11 \\
\vec{\sigma}\end{array}$ & & 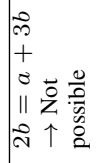 & 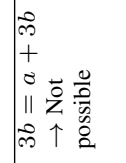 & $\begin{array}{l}\stackrel{8}{N} \\
11 \\
0 \\
\uparrow \\
\uparrow\end{array}$ & & $\begin{array}{l}\stackrel{0}{N} \\
11 \\
0 \\
\uparrow \\
\uparrow\end{array}$ & $\begin{array}{l}0 \\
\infty \\
11 \\
0 \\
\uparrow\end{array}$ \\
\hline$\left|\begin{array}{l}\vec{N} \\
\| 1 \\
\vec{\nabla}\end{array}\right|$ & & 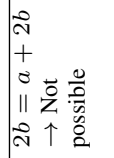 & 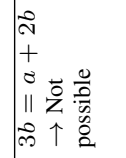 & $\begin{array}{l}\stackrel{8}{N} \\
11 \\
0 \\
\uparrow\end{array}$ & & $\begin{array}{l}\stackrel{0}{N} \\
11 \\
0 \\
\uparrow \\
\uparrow\end{array}$ & $\begin{array}{l}0 \\
\infty \\
11 \\
0 \\
\uparrow\end{array}$ \\
\hline $\begin{array}{l}\infty \\
m \\
+ \\
0 \\
11 \\
0\end{array}$ & & 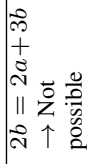 & 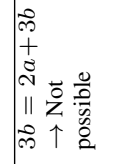 & $\begin{array}{l}0 \\
\stackrel{1}{N} \\
11 \\
0 \\
\uparrow\end{array}$ & & $\begin{array}{l}0 \\
\text { N } \\
11 \\
0 \\
\uparrow \\
\uparrow\end{array}$ & $\begin{array}{l}\infty \\
\infty \\
11 \\
0 \\
\uparrow\end{array}$ \\
\hline 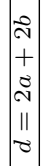 & & 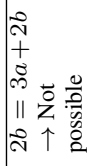 & 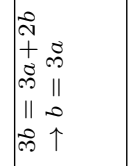 & $\begin{array}{l}\text { in } \\
11 \\
0 \\
\uparrow\end{array}$ & & $\begin{array}{l}\text { i } \\
11 \\
0 \\
\uparrow \\
\uparrow\end{array}$ & $\begin{array}{l}0 \\
\infty \\
11 \\
0 \\
\uparrow\end{array}$ \\
\hline $\begin{array}{l}0 \\
0 \\
+ \\
0 \\
0 \\
11 \\
0 \\
0\end{array}$ & & 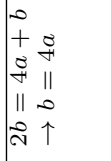 & $\begin{array}{ll}0 & \\
+ & 0 \\
0 & N \\
r & N \\
\| 1 & \| \\
0 & 0 \\
0 & \uparrow\end{array}$ & $\begin{array}{l}0 \\
\text { N } \\
11 \\
0 \\
\uparrow\end{array}$ & & $\begin{array}{l}0 \\
N \\
11 \\
0 \\
\uparrow\end{array}$ & $\begin{array}{l}0 \\
\infty \\
11 \\
0 \\
\uparrow\end{array}$ \\
\hline $\begin{array}{l}\stackrel{0}{N} \\
+ \\
0 \\
11 \\
0\end{array}$ & & 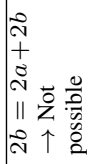 & 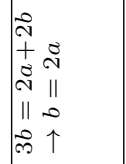 & $\begin{array}{l}0 \\
\text { N } \\
11 \\
0 \\
\uparrow\end{array}$ & & $\begin{array}{l}0 \\
N \\
11 \\
0 \\
\uparrow\end{array}$ & $\begin{array}{l}0 \\
\infty \\
11 \\
0 \\
\uparrow\end{array}$ \\
\hline 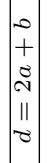 & & 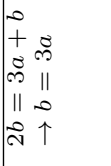 & 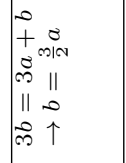 & $\begin{array}{l}0 \\
\text { N } \\
11 \\
0 \\
1 \\
1\end{array}$ & & $\begin{array}{l}0 \\
\text { N } \\
11 \\
0 \\
\uparrow\end{array}$ & $\begin{array}{l}\infty \\
\infty \\
11 \\
0 \\
\uparrow\end{array}$ \\
\hline & 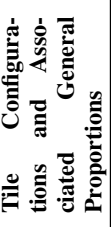 & 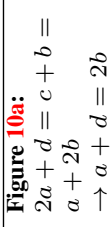 & 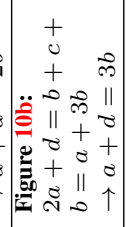 & 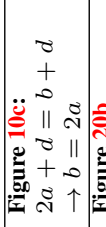 & 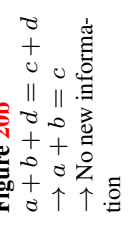 & 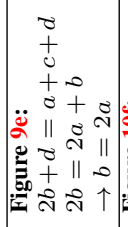 & 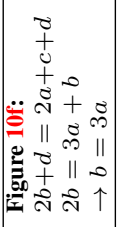 \\
\hline
\end{tabular}

$\frac{0}{2}$ 


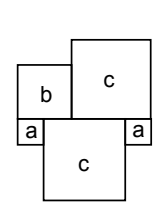

(a)

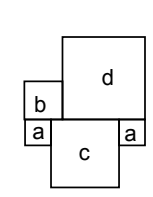

(b)

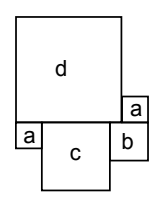

(c)

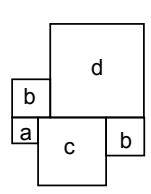

(d)

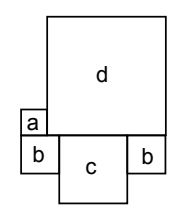

(e)

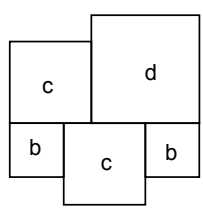

(f)

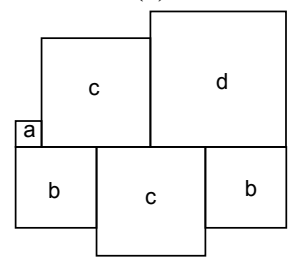

$(\mathrm{g})$

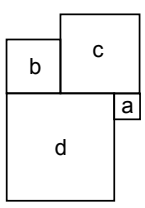

(h)

Figure 9

skeleton of the UET4 tiling $\mathscr{T}$ as in Figure 11:

It is apparent then that some tile or combination of tiles must fit exactly between the dashed lines shown above. A $d$ tile is clearly to large to fit between these lines, and a $c$ tile cannot be placed there by unilaterality. Since $a<b<c$, then some combination of tiles $a$ and $b$ must fit between these lines, and since both $a$ and $b$ must be vortices, then in fact only two tiles may fit between these dashed lines. Because $\mathscr{T}$ is unilateral, it is seen that one $a$ tile and one $b$ tile must fit exactly between the dashed lines in Figure 11. However, this implies that $d=a+b=c<d$, a contradiction. Therefore, there is no tile or combination of tiles that can fit exactly between the dashed lines above, so $c$ must be a vortex.

Next, enumerate possible ways to express $c$ in terms of $a, b$, and $d$ within the $a+b=d$ case. Note that $c<d=a+b$, and observe that when the $c$ tile is surrounded $d$ tiles (as in Figure 12 below), no further specifications as to values of $c$ can be made.

Setting this special case aside momentarily, continue, using the fact that $c$ must be a vortex, to find all possible relationships for $c$ based on implications that arise through each of the three cases found in Figure 13:

Note that in the cases illustrated in $13 \mathrm{a}$ and $13 \mathrm{~b}$, the skeleton of the tiling $\mathscr{T}$ must extend along the dashed lines by virtue of $a, b$, and $c$ all being vortices.

Begin with a statement implying the impossibility of the existence of the partial $c$ corona in Figure 13a in a UET4 tiling.

Lemma 2.4. If $a+b=d$ in a UET4 tiling, then each c corona will not contain an $a$.

Proof. Let $\mathscr{T}$ be a UET4 tiling such that $a+b=d$ and suppose that the $c$ tile's corona contains at least one $a$ tile. Because both $a$ and $c$ are vortices, they must meet at one of their corners as shown in Figure 13a above; the dashed lines show the necessary skeleton extension of $\mathscr{T}$ also required by this vortex condition. Next, determine which tile or combination of tiles can fit exactly between the dashed lines that extend toward the left from the union of the left edges of $a$ and $c$. Were one tile to fill this space, it would have to be a tile $d$, which would imply that $d=a+c>a+b$, a contradiction. Hence more than one tile must fill this space. Were three tiles to fill this space, then the middle tile in the 


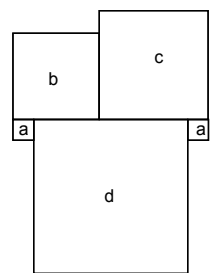

(a)

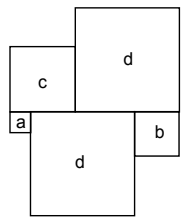

(d)

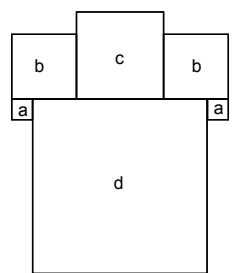

(b)

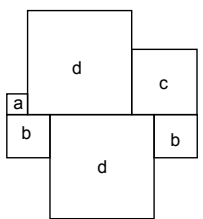

(e)

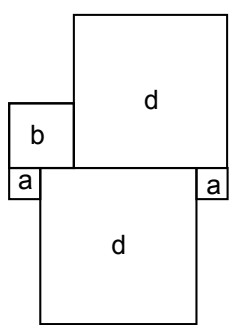

(c)

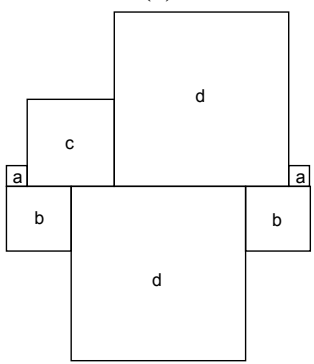

(f)

Figure 10

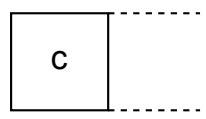

Figure 11: Necessary configuration when $c$ is not a vortex

group must be a non-vortex tile; because $d$ is the only non-vortex tile in this case, one of the three tiles must be a $d$. Then regardless of the other two tiles chosen, the sum of their side lengths will always exceed the length $a+c$ between the dashed lines. Hence three tiles cannot fill this space exactly; it is obvious that four or more tiles similarly cannot fill the space appropriately. This leaves the case where two tiles exactly fill the space between these dashed lines. Then all possible combinations of two distinct tiles are listed as follows: $a$ and $b ; a$ and $c ; a$ and $d ; b$ and $c ; b$ and $d ; c$ and $d$. Of these combinations, the only one that covers the length $a+c$ exactly is the combination $a$ and $c$. Therefore these two tiles must be placed along the left edges of $a$ and $c$ from Figure 13a, and this arrangement is shown below in Figure 14a along with the necessary skeleton extension required by the

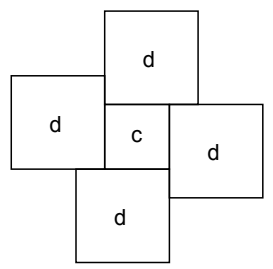

Figure 12: $c$ surrounded by $d$ 's 


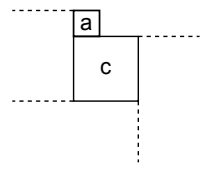

(a)

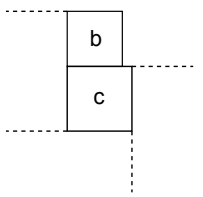

(b)

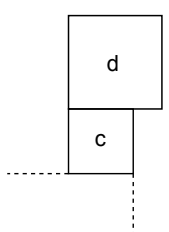

(c)

Figure 13

vortex conditions of $a$ and $c$. The same issue arises again: a tile or group of tiles must fit between the dashed lines that extend downward from the union of the bottom edges of tiles $a_{1}$ and $c$, and by the argument above, only tiles $a$ and $c$ can fill this space exactly. This logic is repeated again in Figure $14 \mathrm{~b}$ to arrive at the partial $c$ corona in Figure 14c, and it is clear by our assumptions that only a tile $c$ can be placed along the top edge of tile $a_{3}$ to complete the corona, which is shown in Figure 14d. By equitransitivity, the tiling that this patch generates is in fact UET2.

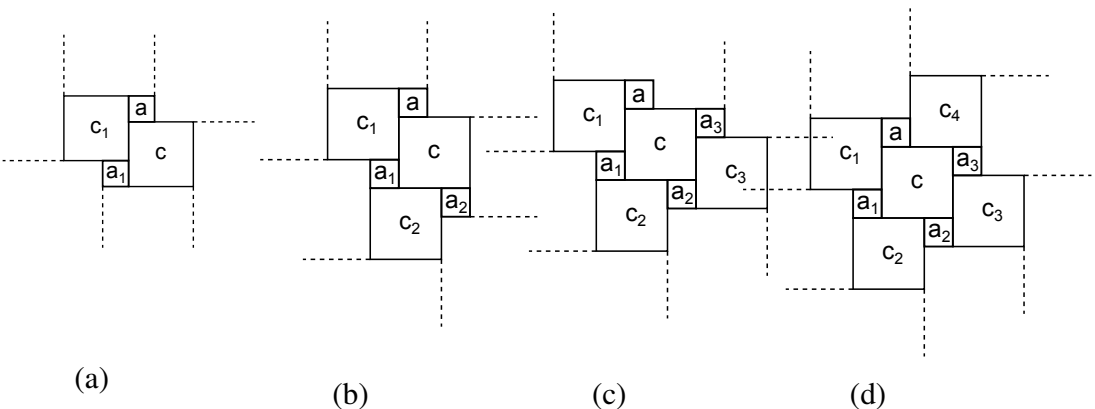

Figure 14: Adjacent $a$ and $c$ tiles results in a UET2 tiling

Having concluded that a $a$ cannot be in the neighborhood of $c$, next consider the specific side lengths for $c$ when $a+b=d$ that arise from the configuration in Figure 13b based on the knowledge that the vortex conditions require that a tile or tiles fit exactly between the dashed lines that extend toward the left of the union of the left edges of $b$ and $c$ in this picture. Note that one tile is too small to fill this space completely, four or more tiles are too large to fill this space completely, and in the case where three tiles exactly fill this space, a non-vortex tile must be in the middle of the group, forcing one of the three tiles to be a $d$ as it is our only non-vortex. Using these facts, the following table enumerates all possibilities where they exist.

Therefore, when $c$ has a tile $b$ in its corona, the side lengths that must be considered are $c=2 a$ and $c=3 a$.

The final case to be considered is that in Figure 13c. Now, because no specific relationships for the side length of $c$ can be established when it is surrounded by only $d$ 's, consider arrangement in Figure 13c where $c$ 's corona contains a tile other than only $d$. Without loss of generality, suppose that this non- $d$ tile can be found along the right edge of $c$ in Figure $13 \mathrm{c}$. It is clear that this tile cannot be a $c$ tile because the resulting tiling would not be 


\begin{tabular}{|l|l|l|}
\hline $\begin{array}{l}\text { All possible combina- } \\
\text { tions of two or three tiles }\end{array}$ & $\begin{array}{l}\text { Can this combination fill } \\
\text { the length in question ex- } \\
\text { actly? }\end{array}$ & $\begin{array}{l}\text { If yes, what does this im- } \\
\text { ply about } c \text { ? }\end{array}$ \\
\hline$a$ and $b$ & no & no \\
\hline$a$ and $c$ & yes & $\begin{array}{l}b+c=a+d=2 a+b \\
\rightarrow c=2 a\end{array}$ \\
\hline$a$ and $d$ & yes & $\begin{array}{l}b+c=b+d \\
\rightarrow \text { no new info }\end{array}$ \\
\hline$b$ and $c$ & no & $\begin{array}{l}b+c=2 a+d=3 a+b \\
\rightarrow c=3 a\end{array}$ \\
\hline$b$ and $d$ & no & \\
\hline$c$ and $d$ & yes & \\
\hline$a$ and $d$ and $a$ & no & \\
\hline$a$ and $d$ and $b$ & no & \\
\hline$a$ and $d$ and $c$ & no & no \\
\hline$b$ and $d$ and $b$ & no & \\
\hline$b$ and $d$ and $c$ & &
\end{tabular}

Table 4

unilateral. Lemma 2.4 implies that this tile cannot be a $a$ tile. Then the non- $d$ tile that must be found in c's corona is a $b$. Knowing that $b$ must be a vortex, there are two arrangements that can result from this, shown in Figure 15:

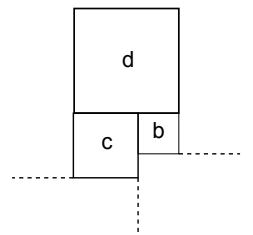

(a)

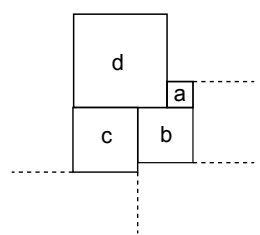

(b)

Figure 15

The arrangement in Figure 15a produces a contradiction because it implies $d=b+c>$ $a+b=d$, so the arrangement in Figure $15 \mathrm{~b}$ is the only viable UET4 possibility. This arrangement implies $b+c=a+d=2 a+b$, so $c=2 a$. Note that this $c$ side length was already found in Table 4 . Therefore, the only $c$ side lengths that must be considered when $a+b=d$ are
1. $c=2 a$
2. $c=3 a$

along with the case in which $c$ 's corona contains only $d$ tiles, in which case the only restriction is given by $b<c<a+b$.

Below is a summary of the side lengths considered when $a$ and $b$ are adjacent. 


\begin{tabular}{|l|l|l|}
\hline \multicolumn{2}{|c|}{$c=a+b$} & \multicolumn{1}{c|}{$d=a+b$} \\
\hline$d$ is not a vortex & \multicolumn{1}{|c|}{$d$ is a vortex } & \\
& & 1. $c=2 a$ \\
1. $d=a+b+c=$ & 1. $d=2 b$ & 2. $c=3 a$ \\
$2 a+2 b$ & 2. $d=3 a$ & \\
2. $d=b+c=a+2 b$ & 3. $d=3 b$ & \\
3. $d=a+c=2 a+b$ & & \\
4. $d=2 b+c=3 b+$ & & \\
$a$ & & \\
5. $d=2 a+c=3 a+$ & & \\
$b$ & & \\
\hline
\end{tabular}

\section{Corona Construction}

With the general UET4 problem having been effectively divided into subcases within which the problem can be appropriately examined, exhaustive lists of all possible coronas for the four sizes of tiles when $a$ and $b$ are adjacent can now be created. The process of constructing all possible coronas for a given case is begun by creating squares of the specified dimensions. Before beginning construction, it should be noted that, for any given tile, there exists at least one edge that extends into the skeleton of the tiling in no more than one direction, meaning it is compatible with the following figure:

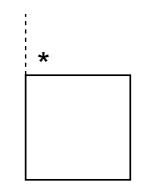

Figure 16

As neighborhoods are constructed, it is assumed that this necessary edge is the top edge of the tile in question.

The illustration of a partial example of corona construction is now presented so as to familiarize the reader with the general process used by examining a specific subcase. In order to illustrate the process used to create all $a, b, c$, and $d$ coronas for a given set of side length proportions, a partial example is now outlined. Consider the case where $a$ and $b$ are adjacent, $c=a+b$, and $d=a+3 b$. The process is illustrated here by constructing all possible $d$ coronas for this case, as these are the most complicated coronas to construct; it should be noted that $c, b$, and $a$ coronas would also need to be constructed for this case. It is known that the arrangement in Figure 16 must appear in any corona, so the tiles that could be placed in the marked corner in that figure are first considered. An $a, b$, or $c$ tile could be placed there, creating three branches shown in Figure 17 that will each be considered in turn. 


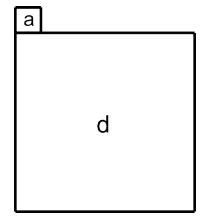

(a)

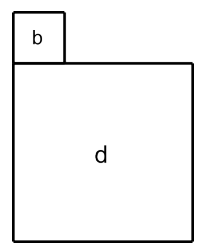

(b)

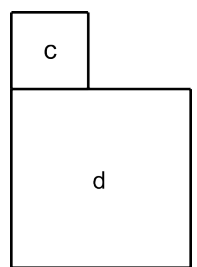

(c)

Figure 17: Three branches to consider for all possible $d$ coronas

Take first the arrangement in Figure 17a; the corona is constructed by placing tiles around this center $d$ in a clockwise direction. As the remaining length along the top edge of $d$ is $3 b$, placing an $a$ or a $b$ next would violate vortex conditions. Hence a $c$ or a $d$ can come next in the corona, creating two new branches shown below.

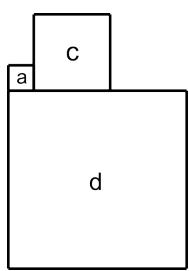

(a)

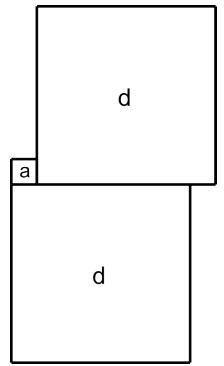

(b)

Figure 18: Two branches from Figure 17a

Consider the arrangement in Figure 18a. The length remaining along the top edge of $d$ is $2 b-a$, so neither an $a$ nor a $b$ can come next due to vortex conditions. A $c$ is also not allowed by unilaterality. A tile $d$ must come next, shown in Figure 19a. Now moving to the right edge of the center $d$ tile, it is evident that a tile $d$ cannot come next by unilaterality; again, vortex conditions say that $a$ nor $b$ can be in this next space either. A tile $c$ must come next, shown in Figure 19b. There is now a distance of $2 b$ along the remaining right edge of the center $d$ tile, so it is again concluded that only a tile $d$ could come next, seen in Figure 19c. Similar logic is employed to conclude that, continuing to move clockwise around the center tile, the remaining sides are covered by a $c$, then a $d$, then a $c$, then a $d$. This arrangement is shown in Figure 19d. However, this contradicts the vortex condition on the $a$ tile, so this arrangement is invalid. Having exhausted all possibilities that could arise from the arrangement in Figure 18a, it is concluded that no viable coronas come from this branch, and attention is next given to the arrangement in Figure 18b. In order to minimize the creation of coronas that are identical up to cyclic permutations of their signature, the arrangement in Figure 18a is henceforth never considered along any edge of the center $d$ tile. 


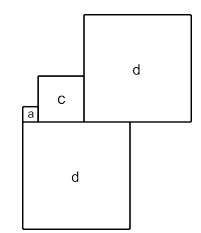

(a)

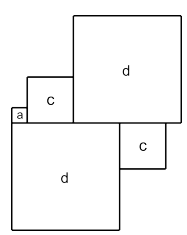

(b)

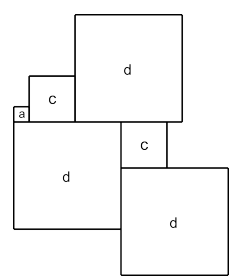

(c)

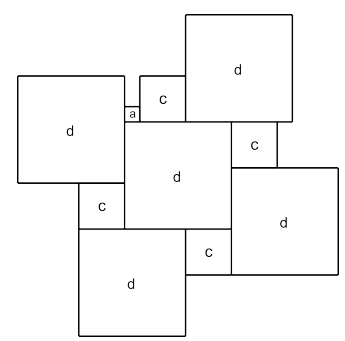

(d)

Figure 19: Successively filling the partial corona in Figure 19a

Corona construction proceeding from the branch in Figure 18b continues along the left edge of the lower $d$ tile, moving clockwise as always. Given this arrangement, it is possible to satisfy vortex conditions if an $a$ or $b$ were placed next in the corona (note that the side length $b=2 a$ is required if this next tile is a $b$; an $a$ is required along the remaining edge of this $b$ in order to make it a vortex). A tile $c$ could come next as well, but a tile $d$ cannot by unilaterality. This leads to three additional branches, shown in Figure 20.

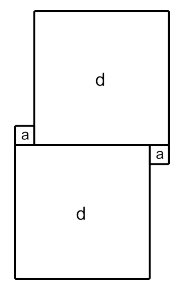

(a)

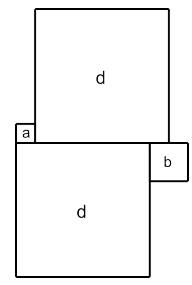

(b)

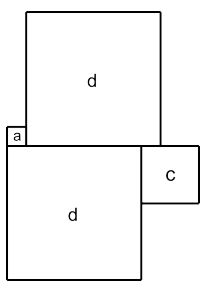

(c)

Figure 20: Three branches from Figure 18b

Consider now the arrangement in Figure 20a. Next, vortex conditions eliminate an $a$ or $b$, so there are two options for the next tile in the corona, namely a $c$ or a $d$ as shown in Figure 21. Recall that the arrangement in Figure 21a contains on its right side a partial corona for which all possibilities were previously exhausted; this branch does not need to be reconsidered.

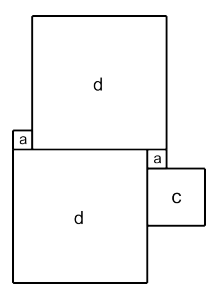

(a)

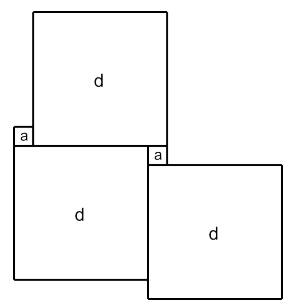

(b)

Figure 21: Two branches from Figure 20a 
Consider the arrangement in Figure 21b. A $d$ tile is the only one that could not come next in the sequence, by unilaterality. The three branches in Figure 22 result (note that the condition $b=2 a$ is again invoked in Figure 22b).

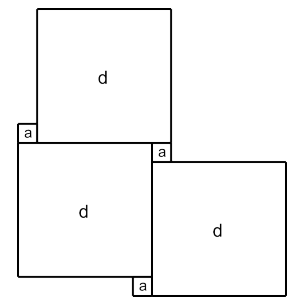

(a)

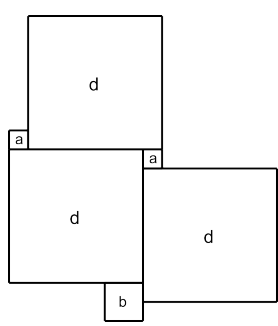

(b)

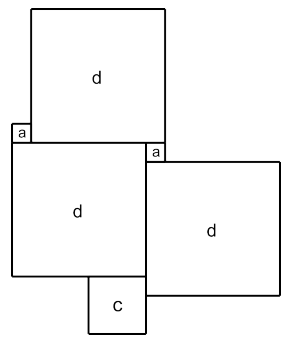

(c)

Figure 22: Three branches from Figure 21b

Consider the arrangement in Figure 22a. In choosing the next tile, vortex conditions eliminate a or b, so $c$ or $d$ could come next in the corona, but as placing a $c$ in this position would lead to a cyclic permutation of an arrangement previously exhausted, only the case where a $d$ comes next is considered. Following this $d$ (seen in Figure 23a), a tile $a, b$, or $c$ could be placed ( $d$ being disallowed by unilaterality). These three branches are shown in Figures 23b, 23c, and 23d.

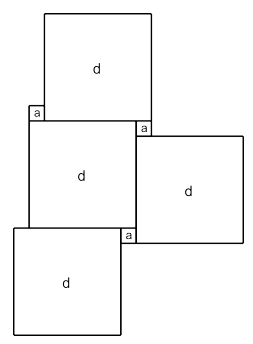

(a)

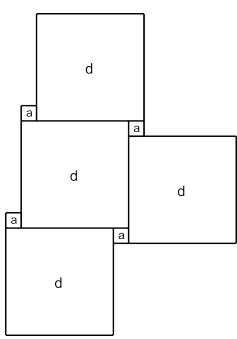

(b)

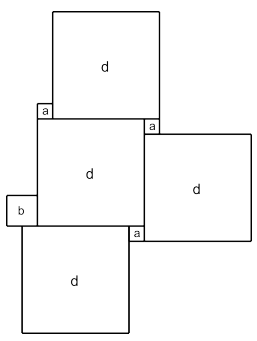

(c)

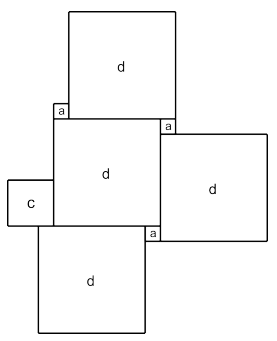

(d)

Figure 23: Partial corona a.d.a.d.a.d and three resulting branches

As placing a $c$ next in the partial corona of Figure $23 \mathrm{~b}$ has been shown to be an impossible arrangement (see the branch from Figure 18a), only a $d$ could complete this corona appropriately. This gives the complete corona signature a.d.a.d.a.d.a.d. Next, the vortex condition on the first $a$ tile in Figure 23c would require that a combination of tiles fit exactly along the remaining distance $2 a+2 b$ along the left edge. Tile lengths require that this include at least one $d$ tile, which would violate the vortex conditions. Hence the arrangement in Figure 23c does not lead to a viable corona. Finally, the partial corona of Figure $23 \mathrm{~d}$ can only be completed with a $d$, again violating the vortex condition on $a$; no viable coronas result from this arrangement. Now, all arrangements branching from that in Figure $23 \mathrm{a}$ have been exhausted. Figure $22 \mathrm{~b}$ is the branch that should be returned to next.

The partial example outlined in this section is illustrated by a tree diagram in Figure 24; this shows branches of all possibilities considered along with which branches produce 
viable UET4 coronas and which are unfruitful. The tree diagram shows all branches that arise from placing an $a$ tile in the asterisked position in Figure 16; those arising from placing a $b$ or $c$ in that position are not included in the diagram for brevity's sake.

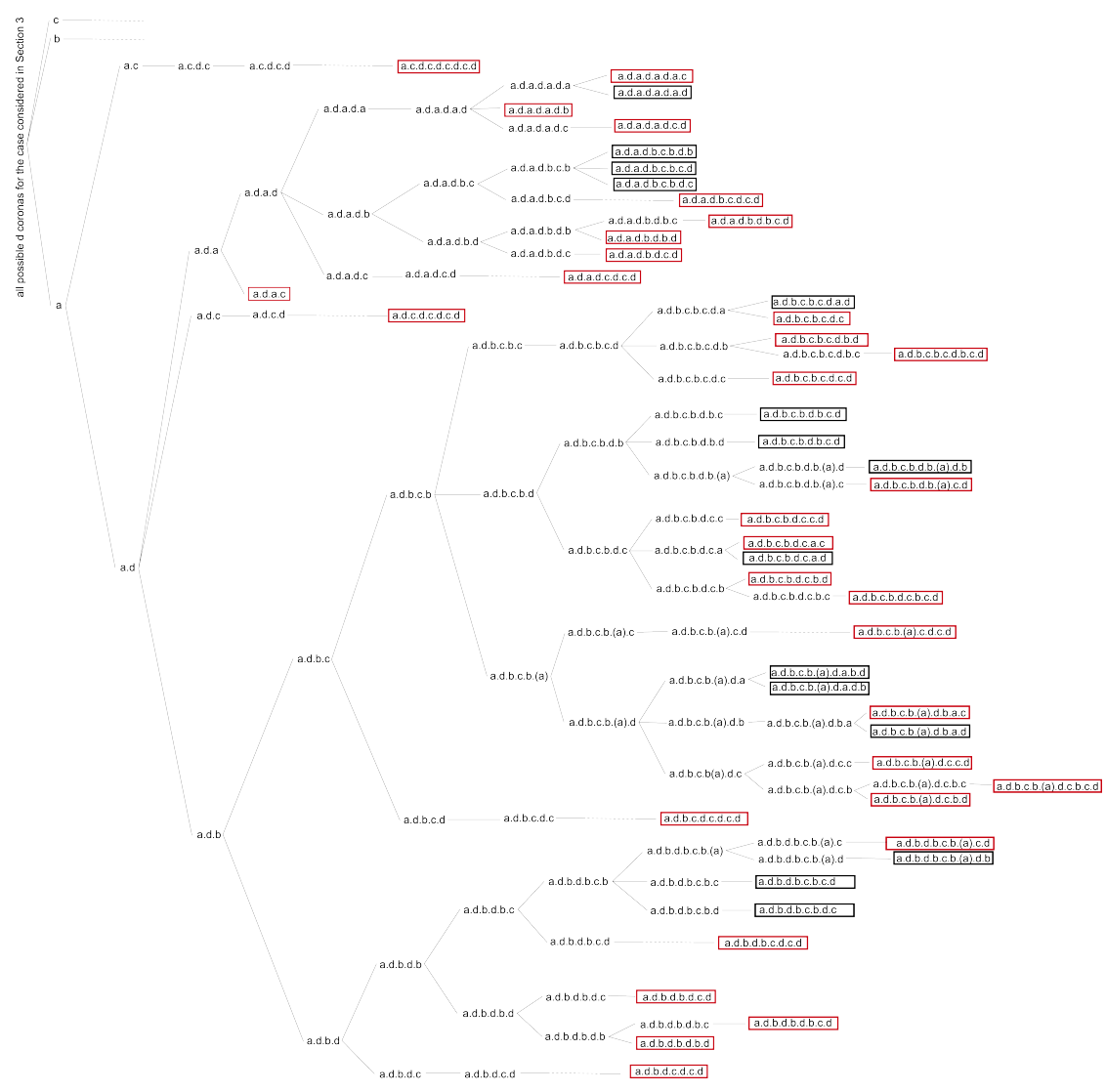

Figure 24: A tree diagram to accompany the illustration of corona construction found in Section 4. All $d$ corona possibilities illustrated in Section 4 are pictured here; those omitted are not featured here either. Coronas in black rectangles are viable UET4 coronas; those in red rectangles (full or partial) do not lead to viable UET4 coronas. The dotted lines mark where a step or series of steps (for each of which only one possible tile could appear next) in the construction process have been omitted for brevity's sake.

\section{Construction of the UET4 Tilings from Viable Coronas}

Having compiled a list of all possible $a, b, c$, and $d$ coronas for each of the cases when $a$ and $b$ are adjacent, it remains to determine which combinations of these coronas can be combined to generate a UET4 tiling. The extension of Schattschneider's method of finding coronas that correspond to a tiling is illustrated with an example. Consider the case where $a$ and $b$ are adjacent, $c=a+b$, and $d=3 a$. Using the process explained earlier, the corresponding set of viable coronas are: 


\begin{tabular}{|c|c|c|c|}
\hline$a$ coronas & $b$ coronas & $c$ coronas & $d$ coronas \\
\hline $\begin{array}{l}b . b . c . b \\
\text { c.b.c.b } \\
\text { b.b.c.c } \\
\text { c.b.c.d } \\
\text { c.b.c.c } \\
\text { d.b.c.d } \\
\text { d.c.d.d } \\
\text { d.d.c.c } \\
\text { c.d.c.d } \\
\text { c.d.c.c } \\
\text { c.c.c.c } \\
\text { d.d.d.d }\end{array}$ & $\begin{array}{l}a . b . a . b . a . b . a . b \\
\text { a.b.a.b.a.c.c } \\
\text { a.b.a.b.a.d.c } \\
\text { a.b.a.c.c.c } \\
\text { a.b.a.c.d.c } \\
\text { a.b.a.d.c.c } \\
\text { a.b.a.d.d.c } \\
\text { a.c.c.a.c.c } \\
\text { a.c.c.a.d.c } \\
\text { a.c.c.c.c } \\
\text { a.c.c.d.c } \\
\text { a.c.c.d.c } \\
\text { a.c.d.c.a.b } \\
\text { a.c.d.c.c } \\
\text { a.c.d.d.c } \\
\text { a.d.c.a.b.a.b } \\
\text { a.d.c.a.c.c } \\
\text { a.d.c.a.d.c } \\
\text { a.d.c.c.a.b } \\
\text { a.d.c.c.c } \\
\text { a.d.c.d.c } \\
\text { a.d.d.c.a.b } \\
\text { a.d.d.c.c } \\
\text { a.d.d.d.c } \\
\text { c.c.c.c } \\
\text { c.c.c.d } \\
\text { c.d.c.d } \\
\text { c.c.d.d } \\
\text { c.d.d.d } \\
\text { d.d.d.d }\end{array}$ & $\begin{array}{l}a . b . c . b . a . b . c . b \\
\text { a.b.c.b.a.c.a.c } \\
\text { a.b.c.b.c.b.c.b } \\
\text { a.c.a.c.a.c.a.c } \\
\text { b.c.b.c.b.c.b.c } \\
\text { d.d.d.d } \\
\text { a.b.d.c.d.a.c } \\
\text { a.b.d.d.c.a.c } \\
\text { a.b.d.d.d.c } \\
\text { a.b.d.c.a.b.d } \\
\text { a.b.d.d.a.b.d } \\
\text { a.b.d.d.c.d } \\
\text { a.b.c.b.c.b.a.c } \\
\text { a.b.c.b.d.c.b } \\
\text { a.b.c.b.d.d.b } \\
\text { a.b.c.b.d.d.c } \\
\text { a.b.c.b.d.c.d } \\
\text { a.b.c.b.d.a.b.d } \\
\text { a.b.c.b.d.a.d.b } \\
\text { a.b.c.d.b.a.d.b } \\
\text { a.b.c.d.b.a.b.d } \\
\text { a.b.d.a.b.d.a.c } \\
\text { a.b.d.a.c.a.b.d } \\
\text { a.b.d.a.c.a.d.b } \\
\text { a.b.d.a.d.b.a.c } \\
\text { a.b.d.c.b.a.b.d } \\
\text { a.b.d.c.b.a.d.b } \\
\text { b.c.b.c.b.c.d } \\
\text { b.c.b.c.b.d.d } \\
\text { b.c.b.c.d.d } \\
\text { b.c.b.d.d.d } \\
\text { b.c.d.b.c.d } \\
\text { b.c.d.b.d.d } \\
\text { b.c.d.d.d } \\
\text { b.d.d.b.d.d } \\
\text { b.d.d.d.d } \\
\text { a.b.d.b.a.b.d } \\
\text { a.b.d.b.a.d.b }\end{array}$ & $\begin{array}{l}\text { b.d.b.d.b.d.b.d } \\
\text { c.d.c.d.c.d.c.d } \\
\text { a.d.a.d.a.d.a.d } \\
\text { a.d.a.d.a.d.a.d } \\
\text { a.c.b.c.a.c.b.c } \\
\text { a.c.b.c.a.c.c.b } \\
\text { a.c.b.c.a.d.c.b } \\
\text { a.c.b.c.c.b.a.d } \\
\text { a.c.b.c.c.b.a.d } \\
\text { a.c.b.c.c.d.c.b } \\
\text { a.c.b.c.c.d.c.b } \\
\text { a.c.b.c.c.d.c.b } \\
\text { a.c.b.d.b.c.a.d } \\
\text { a.c.b.d.b.c.c.b } \\
\text { a.c.b.d.b.d.c.b } \\
\text { a.c.b.d.b.d.b.c } \\
\text { a.c.b.d.c.b.a.d } \\
\text { a.c.b.d.c.d.c.b } \\
\text { a.c.c.b.a.c.b.c } \\
\text { a.c.c.b.a.c.c.b } \\
\text { a.c.c.b.a.d.a.d } \\
\text { a.c.c.b.a.d.c.b } \\
\text { a.c.c.d.c.b.a.d } \\
\text { a.c.c.d.c.d.c.b } \\
\text { a.d.a.d.a.d.c.b } \\
\text { a.d.a.d.c.d.c.b } \\
\text { a.d.c.b.a.d.c.b } \\
\text { a.d.c.d.c.b.a.d } \\
\text { a.d.c.d.c.d.c.b }\end{array}$ \\
\hline
\end{tabular}

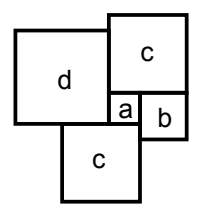

Figure 25: An a corona with signature c.b.c.d.

Choose the $a$ corona c.b.c.d, as illustrated in Figure 25. Observe that because of the tiles neighboring the $b$ tile in Figure 25, and because $\mathscr{T}$ is equitransitive, the $b$ corona for 
$\mathscr{T}$ must contain the partial corona c.a.c. Likewise, the $c$ corona for $\mathscr{T}$ must contain the partial coronas b.a.d and d.a.b and the $d$ corona for $\mathscr{T}$ must contain the partial corona c.a.c. Finding the partial $b$ coronas that are compatible with our choice of $a$ corona is easily automated. For example, to find the first partial $b$ corona, find the first instance of $b$ in the $a$ corona, c.b.c.d. A partial corona signature for $b$ would then be the letter cyclically preceeding this instance of $b$, followed by $a$ (since this partial signature is taken from an $a$ corona signature), followed by the letter cyclically following this instance of $b$, yielding the partial $b$ corona signature c.a.c. This process can be repeated for each occurrence of $b$ and for $c$ and $d$ as well. Note that partial corona signatures that are cyclic permutations of each other are considered equivalent, and so are reverse orderings. Performing such a search for partial $b, c$, and $d$ corona signatures corresponding to the initial choice of $a$ corona signature, c.b.c.d, yields the following.

\begin{tabular}{|l|l|l|l|}
\hline A & B & C & D \\
\hline$a$ corona & Partial $b$ coronas & Partial $c$ coronas & Partial $d$ coronas \\
\hline c.b.c.d & c.a.c & b.a.d & c.a.c \\
\hline
\end{tabular}

Search the list of full $b$ coronas for any which contain the partial corona c.a.c. In this example, after the search is performed, the five matching $b$ coronas include a.c.c.a.c.c, a.c.c.a.d.c, a.c.c.c.c, a.c.c.d.c and a.c.d.d.c. Use these coronas to create five corresponding 2-tuples of compatible $a$ and $b$ corona signatures (e.g. (c.b.c.d, a.c.c.a.c.c), (c.b.c.d, a.c.c.a.d.c), etc). For each new 2-tuple $(x, y)$, add the $b$ corona's corresponding partial $a$, $c$ and $d$ coronas to their respective columns as in Table 5.

\begin{tabular}{|c|c|c|c|c|c|}
\hline & $\mathbf{A}$ & B & C & D & $\mathbf{E}$ \\
\hline & $\begin{array}{l}(x, y) \\
\text { Tuple }\end{array}$ & $\begin{array}{l}\text { partial a } \\
\text { coronas in } \\
(x, y)\end{array}$ & $\begin{array}{l}\text { partial } b \\
\text { coronas in } \\
(x, y)\end{array}$ & $\begin{array}{l}\text { partial } \quad c \\
\text { coronas in } \\
(x, y)\end{array}$ & $\begin{array}{l}\text { partial d } \\
\text { coronas in } \\
(x, y)\end{array}$ \\
\hline 1 & $\begin{array}{l}\text { (c.b.c.d, } \\
\text { a.c.c.a.c.c) }\end{array}$ & c.b.c & c.a.c & $\begin{array}{l}\text { a.b.c } \\
\text { b.a.d }\end{array}$ & c.a.c \\
\hline 2 & $\begin{array}{l}\text { c.b.c.d, } \\
\text { a.c.c.a.d.c) }\end{array}$ & $\begin{array}{l}\text { c.b.c } \\
\text { c.b.d }\end{array}$ & c.a.c & $\begin{array}{l}d . b . a \\
\text { a.b.c } \\
\text { b.a.d }\end{array}$ & $\begin{array}{l}a . b . c \\
c . a . c\end{array}$ \\
\hline 3 & $\begin{array}{l}(c . b . c . d, \\
a . c . c . c . c)\end{array}$ & c.b.c & c.a.c & $\begin{array}{l}a . b . c \\
\text { c.b.c } \\
\text { b.a.d }\end{array}$ & c.a.c \\
\hline 4 & $\begin{array}{l}(c . b . c . d, \\
a . c . c . d . c)\end{array}$ & c.b.c & c.a.c & $\begin{array}{l}\text { a.b.c } \\
\text { c.b.d } \\
\text { d.b.a } \\
\text { b.a.d }\end{array}$ & $\begin{array}{l}c . b . c \\
c . a . c\end{array}$ \\
\hline 5 & $\begin{array}{l}\text { (c.b.c.d, } \\
\text { a.c.d.d.c) }\end{array}$ & c.b.c & c.a.c & $\begin{array}{l}\text { a.b.d } \\
\text { b.a.d }\end{array}$ & $\begin{array}{l}\text { c.b.d } \\
\text { c.a.c }\end{array}$ \\
\hline
\end{tabular}

Table 5

While each of the $b$ coronas in $(x, y)$ contain the necessary partial $b$ corona signature c.a.c, some may contain other partial $a$ signatures that are not compatible with the original choice of $a$ signature in $(x, y)$. For example, the $b$ corona of $(x, y)$ in line 2, a.c.c.a.d.c, 
has the partial $a$ corona $c . b . d$, which is not contained in the original $a$ corona, c.b.c.d. So Line 2 is eliminated from further consideration.

Next, for each surviving tuple $(x, y)$ in Table 5 , search the list of full $c$ coronas for those that contain the tuple's corresponding partial $c$ coronas. For example, the tuple in line 4 has partial $c$ coronas a.b.d, d.b.c, c.b.a and b.a.d. All of these partial coronas are contained only in full $c$ corona a.b.c.b.d.a.b.d. For each full $c$ corona that is compatible with our 2-tuple $(x, y)$, create a new 3-tuple $(x, y, z)$ by appending compatible $c$ corona signature, as in column A of Table 6. Also, list the partial $a, b, c$, and $d$ coronas that are contained in $(x, y, z)$ (columns $\mathbf{B}$ - E).

There are now nineteen viable $(x, y, z)$ tuples. For each of these 3 -tuples, check if all of the partial $a$ and $b$ coronas are contained in the full $a$ and $b$ coronas in the tuple. If not, remove that tuple. For example, consider the 3-tuple (c.b.c.d,a.c.c.a.c.c,a.b.c.b.d.c.d) of Line 4. a.c.d is listed as a partial $b$ corona, but a.c.d is not contained in this tuple's full $b$ corona, which is a.c.c.a.c.c. Therefore, delete line 4. After performing this check for all of the tuples, there are only three tuples which pass the test, shown in Table 7.

Finally, search the list of full $d$ coronas that contain all of the partial $d$ coronas for each $(x, y, z)$ 3-tuple to create a new list of 4-tuples $(x, y, z, w)$ where $w$ is a $d$ corona that is compatible with the 3 -tuple $(x, y, z)$. For this example, this is Column $A$ of Table 8 . Add to this table columns containing the partial $a, b, c$, and $d$ coronas contained in $(x, y, z, w)$ which will be used to check the viability of $(x, y, z, w)$ as before.

For each tuple, check if all of the partial $a, b$ and $c$ coronas are contained in the full $a, b$ and $c$ coronas in the tuple. For the tuple in line 1, this is not the case. Partial $a$ corona b.d.c is not contained in full $a$ corona c.b.c.d. The tuple in line 2 passes the test.

At this point, from our original conditions and choice of $a$ corona signature, there remains only one combination of $a, b, c$, and $d$ coronas that may result in a tiling or tilings. When this process is automated and performed for all possible cases (e.g. $2 a+b=d$ )) and choices of $a$ corona signatures, the following list of 4-tuples $(x, y, z, w)$ is generated.

The process of constructing a tiling is demonstrated using a 4-tuple from Table 9. Consider the 4-tuple (d.b.c.d, a.d.d.c.c, a.b.c.b.d.c.d, a.c.c.b.d.b.a.d), for which $b=2 a$, $c=a+b$, and $d=2 a+b$. It is known that $b=2 a$ because it is a necessary condition for a $c$ tile to have the corona a.b.c.b.d.c.d. The tiles and their coronas are displayed in Figure 26.
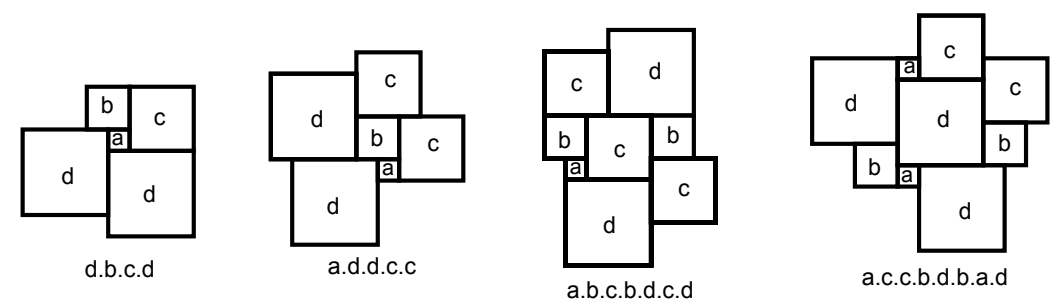

Figure 26: (d.b.c.d, a.d.d.c.c, a.b.c.b.d.c.d, a.c.c.b.d.b.a.d), for which $b=2 a, c=a+b$ and $d=2 a+b$

There are many ways to construct a tiling. The end goal is to create a patch that will tile the plane. For example, start with the $d$ tile and its corona. Complete the coronas of the tiles which surround the $d$ using reflections and rotations of the coronas in Figure 26. This 


\begin{tabular}{|c|c|c|c|c|c|}
\hline & $\mathbf{A}$ & B & C & D & $\mathbf{E}$ \\
\hline & $(x, y, z)$ Tuple & $\begin{array}{l}\text { partial } a \text { coronas in } \\
(x, y, z)\end{array}$ & $\begin{array}{l}\text { partial } b \text { coronas in } \\
(x, y, z)\end{array}$ & $\begin{array}{l}\text { partial } c \text { coronas in } \\
(x, y, z)\end{array}$ & $\begin{array}{l}\text { partial } d \text { coronas in } \\
(x, y, z)\end{array}$ \\
\hline 1 & $\begin{array}{l}\text { (c.b.c.d, a.c.c.a.c.c, } \\
\text { a.b.c.d.b.a.b.d) }\end{array}$ & $\begin{array}{l}\text { b.c.d } \\
\text { b.c.b } \\
\text { c.b.c }\end{array}$ & $\begin{array}{l}\text { c.c.a } \\
\text { a.c.d } \\
\text { c.a.c }\end{array}$ & $\begin{array}{l}\text { a.b.c } \\
\text { b.a.d }\end{array}$ & $\begin{array}{l}\text { a.c.b } \\
\text { c.c.b } \\
\text { c.a.c }\end{array}$ \\
\hline 2 & $\begin{array}{l}\text { (c.b.c.d, a.c.c.a.c.c, } \\
\text { a.b.d.c.b.a.d.b) }\end{array}$ & $\begin{array}{l}\text { b.c.b } \\
\text { b.c.d } \\
\text { c.b.c }\end{array}$ & $\begin{array}{l}\text { a.c.c.c } \\
\text { a.c.d } \\
\text { c.a.c }\end{array}$ & $\begin{array}{l}\text { a.b.c } \\
\text { b.a.d }\end{array}$ & $\begin{array}{l}\text { c.c.b } \\
\text { a.c.b } \\
\text { c.a.c }\end{array}$ \\
\hline 3 & $\begin{array}{l}\text { (c.b.c.d, a.c.c.a.c.c, } \\
\text { a.b.c.d.b.a.d.b) }\end{array}$ & $\begin{array}{l}b c b \\
b . c . d \\
c . b . c\end{array}$ & $\begin{array}{l}\text { a.c.d } \\
\text { a.c.c } \\
\text { c.a.c }\end{array}$ & $\begin{array}{l}a . b . c \\
\text { b.a.d }\end{array}$ & $\begin{array}{l}\text { b.c.c. } \\
\text { a.c.b } \\
\text { c.a.c }\end{array}$ \\
\hline 4 & $\begin{array}{l}\text { (c.b.c.d, a.c.c.a.c.c, } \\
\text { a.b.c.b.d.c.d) }\end{array}$ & $\begin{array}{l}\text { d.c.b } \\
\text { c.b.c }\end{array}$ & $\begin{array}{l}\text { c.c.a } \\
\text { a.c.d } \\
\text { c.a.c }\end{array}$ & $\begin{array}{l}\text { a.b.c } \\
\text { b.a.d }\end{array}$ & $\begin{array}{l}\text { a.c.b } \\
\text { b.c.c } \\
\text { c.a.c }\end{array}$ \\
\hline 5 & $\begin{array}{l}\text { (c.b.c.d, a.c.c.a.c.c, } \\
\text { a.b.d.c.b.a.b.d) }\end{array}$ & $\begin{array}{l}\text { d.c.b } \\
\text { b.c.b } \\
\text { c.b.c }\end{array}$ & $\begin{array}{l}\text { c.c.a } \\
\text { a.c.d } \\
\text { c.a.c }\end{array}$ & $\begin{array}{l}a . b . c \\
\text { b.a.d }\end{array}$ & $\begin{array}{l}\text { b.c.a } \\
\text { b.c.c } \\
\text { c.a.c }\end{array}$ \\
\hline 6 & $\begin{array}{l}\text { (c.b.c.d, a.c.c.a.c.c, } \\
\text { a.b.c.b.d.a.b.d })\end{array}$ & $\begin{array}{l}\text { d.c.b } \\
\text { c.b.c }\end{array}$ & $\begin{array}{l}\text { a.c.d } \\
\text { a.c.c } \\
\text { c.c.d } \\
\text { c.a.c }\end{array}$ & $\begin{array}{l}a . b . c \\
\text { b.a.d }\end{array}$ & $\begin{array}{l}\text { b.c.a } \\
\text { c.a.c }\end{array}$ \\
\hline 7 & $\begin{array}{l}\text { (c.b.c.d, a.c.c.d.c, } \\
\text { a.b.c.b.d.a.b.d })\end{array}$ & $\begin{array}{l}\text { d.c.b } \\
\text { c.b.c }\end{array}$ & $\begin{array}{l}\text { a.c.d } \\
\text { a.c.c } \\
\text { c.c.d } \\
\text { c.a.c }\end{array}$ & $\begin{array}{l}\text { a.b.c } \\
\text { c.b.d } \\
\text { d.b.a } \\
\text { b.a.d }\end{array}$ & $\begin{array}{l}\text { b.c.a } \\
\text { c.b.c } \\
\text { c.a.c }\end{array}$ \\
\hline 8 & $\begin{array}{l}\text { (c.b.c.d, a.c.d.d.c, } \\
\text { a.b.c.d.b.a.d.b) }\end{array}$ & $\begin{array}{l}\text { b.c.b } \\
\text { b.c.d } \\
\text { c.b.c }\end{array}$ & $\begin{array}{l}\text { a.b.d } \\
\text { b.a.d }\end{array}$ & $\begin{array}{l}\text { a.c.d } \\
\text { a.c.c }\end{array}$ & $\begin{array}{l}\text { b.c.c } \\
\text { a.c.b } \\
\text { c.b.d } \\
\text { c.a.c }\end{array}$ \\
\hline 9 & $\begin{array}{l}\text { (c.b.c.d, a.c.d.d.c, } \\
\text { a.b.d.d.c.d })\end{array}$ & $\begin{array}{l}\text { d.c.b } \\
\text { c.b.c }\end{array}$ & $\begin{array}{l}a . b . d \\
\text { b.a.d }\end{array}$ & a.c.d & $\begin{array}{l}a . c . c \\
\text { d.c.c } \\
\text { b.c.d } \\
\text { c.b.d } \\
\text { c.a.c }\end{array}$ \\
\hline 10 & $\begin{array}{l}\text { (c.b.c.d, a.c.d.d.c, } \\
\text { a.b.d.b.a.b.d) }\end{array}$ & $\begin{array}{l}\text { b.c.d } \\
\text { b.c.b } \\
\text { c.b.c }\end{array}$ & $\begin{array}{l}\text { a.b.d } \\
\text { b.a.d }\end{array}$ & a.c.d & $\begin{array}{l}a . c . b \\
\text { b.c.b } \\
\text { c.b.d } \\
\text { c.a.c }\end{array}$ \\
\hline 11 & $\begin{array}{l}\text { (c.b.c.d, a.c.d.d.c, } \\
\text { a.b.d.c.b.a.b.d) }\end{array}$ & $\begin{array}{l}\text { b.c.d } \\
\text { b.c.b } \\
\text { c.b.c }\end{array}$ & $\begin{array}{l}\text { a.b.d } \\
\text { b.a.d }\end{array}$ & $\begin{array}{l}\text { a.c.c } \\
\text { a.c.d }\end{array}$ & $\begin{array}{l}a . c . b \\
\text { b.c.c } \\
\text { c.b.d } \\
\text { c.a.c }\end{array}$ \\
\hline 12 & $\begin{array}{l}\text { (c.b.c.d, a.c.d.d.c, } \\
\text { a.b.d.d.a.b.d) }\end{array}$ & $\begin{array}{l}\text { b.c.d } \\
\text { c.b.c }\end{array}$ & $\begin{array}{l}\text { a.b.d } \\
\text { b.a.d }\end{array}$ & a.c.d & $\begin{array}{l}\text { a.c.d } \\
\text { b.c.d } \\
\text { c.b.d } \\
\text { c.a.c }\end{array}$ \\
\hline 13 & $\begin{array}{l}\text { (c.b.c.d, a.c.d.d.c, } \\
\text { a.b.d.c.a.b.d) }\end{array}$ & $\begin{array}{l}\text { b.c.d } \\
\text { c.c.b } \\
\text { c.b.c }\end{array}$ & $\begin{array}{l}\text { a.b.d } \\
\text { b.a.d }\end{array}$ & a.c.d & $\begin{array}{l}a . c . b \\
\text { b.c.c } \\
\text { c.b.d } \\
\text { c.a.c }\end{array}$ \\
\hline 14 & $\begin{array}{l}\text { (c.b.c.d, a.c.d.d.c, } \\
\text { a.b.d.c.b.a.d.b) }\end{array}$ & $\begin{array}{l}\text { b.c.b } \\
\text { b.c.d } \\
\text { c.b.c.c }\end{array}$ & $\begin{array}{l}\text { a.b.d } \\
\text { b.a.d }\end{array}$ & $\begin{array}{l}\text { a.c.c } \\
\text { a.c.d }\end{array}$ & $\begin{array}{l}\text { c.c.b } \\
\text { a.c.b } \\
\text { c.b.d } \\
\text { c.a.c }\end{array}$ \\
\hline 15 & $\begin{array}{l}\text { (c.b.c.d, a.c.d.d.c, } \\
\text { a.b.d.b.a.d.b) }\end{array}$ & $\begin{array}{l}\text { b.c.b } \\
\text { b.c.d } \\
\text { c.b.c.c }\end{array}$ & $\begin{array}{l}a . b . d \\
\text { b.a.d }\end{array}$ & a.c.d & $\begin{array}{l}\text { b.c.b } \\
\text { a.c.b } \\
\text { c.b.d } \\
\text { c.a.c }\end{array}$ \\
\hline 16 & $\begin{array}{l}\text { (c.b.c.d, a.c.d.d.c, } \\
\text { a.b.d.a.c.a.b.d) }\end{array}$ & $\begin{array}{l}\text { b.c.d } \\
\text { d.c.c } \\
\text { c.c.b } \\
\text { c.b.c }\end{array}$ & $\begin{array}{l}\text { a.b.d } \\
\text { b.a.d }\end{array}$ & a.c.d & $\begin{array}{l}\text { b.c.a } \\
\text { c.b.d } \\
\text { c.a.c }\end{array}$ \\
\hline 17 & $\begin{array}{l}\text { (c.b.c.d, a.c.d.d.c, } \\
\text { a.b.c.b.d.a.b.d })\end{array}$ & $\begin{array}{l}\text { d.c.b } \\
\text { c.b.c }\end{array}$ & $\begin{array}{l}\text { a.b.d } \\
\text { b.a.d }\end{array}$ & $\begin{array}{l}\text { d.c.a } \\
\text { a.c.c } \\
\text { c.c.d }\end{array}$ & $\begin{array}{l}\text { b.c.a } \\
\text { c.b.d } \\
\text { c.a.c }\end{array}$ \\
\hline 18 & $\begin{array}{l}\text { (c.b.c.d, a.c.d.d.c, } \\
\text { a.b.c.d.b.a.b.d })\end{array}$ & $\begin{array}{l}\text { b.c.d } \\
\text { b.c.b } \\
\text { c.b.c }\end{array}$ & $\begin{array}{l}\text { a.b.d } \\
\text { b.a.d }\end{array}$ & $\begin{array}{l}\text { a.c.d } \\
\text { a.c.c }\end{array}$ & $\begin{array}{l}\text { a.c.b } \\
\text { c.c.b } \\
\text { c.b.d } \\
\text { c.a.c }\end{array}$ \\
\hline 19 & $\begin{array}{l}\text { (c.b.c.d, a.c.d.d.c, } \\
\text { a.b.d.a.b.d.a.c) }\end{array}$ & $\begin{array}{l}\text { b.c.d } \\
\text { d.c.c } \\
\text { c.c.b } \\
\text { c.b.c }\end{array}$ & $\begin{array}{l}\text { a.b.d } \\
\text { b.a.d }\end{array}$ & a.c.d & $\begin{array}{l}\text { b.c.a } \\
\text { c.b.d } \\
\text { c.a.c.c }\end{array}$ \\
\hline
\end{tabular}

Table 6 


\begin{tabular}{|c|c|c|c|c|c|}
\hline & $\mathbf{A}$ & B & $\mathbf{C}$ & D & $\mathbf{E}$ \\
\hline & $(x, y, z)$ Tuple & $\begin{array}{l}\text { partial } a \text { coro- } \\
\text { nas in }(x, y, z)\end{array}$ & $\begin{array}{l}\text { partial } b \text { coro- } \\
\text { nas in }(x, y, z)\end{array}$ & $\begin{array}{l}\text { partial } c \text { coro- } \\
\text { nas in }(x, y, z)\end{array}$ & $\begin{array}{l}\text { partial } d \text { coro- } \\
\text { nas in }(x, y, z)\end{array}$ \\
\hline 1 & $\begin{array}{l}(c . b \cdot c \cdot d, \\
a \cdot c \cdot c \cdot d \cdot c, \\
a . b \cdot c \cdot b \cdot d \cdot a \cdot b \cdot d)\end{array}$ & $\begin{array}{l}\text { d.c.b } \\
\text { c.b.c }\end{array}$ & $\begin{array}{l}\text { a.c.d } \\
\text { a.c.c } \\
\text { c.c.d } \\
\text { c.a.c }\end{array}$ & $\begin{array}{l}a . b . c \\
\text { c.b.d } \\
\text { d.b.a } \\
\text { b.a.d }\end{array}$ & $\begin{array}{l}\text { b.c.a } \\
\text { c.b.c } \\
\text { c.a.c }\end{array}$ \\
\hline 2 & $\begin{array}{l}\text { (c.b.c.d, } \\
a . c . d . d . c, \\
a . b . d . d . c . d)\end{array}$ & $\begin{array}{l}\text { d.c.b } \\
\text { c.b.c }\end{array}$ & $\begin{array}{l}\text { a.b.d } \\
\text { b.a.d }\end{array}$ & a.c.d & $\begin{array}{l}\text { a.c.c } \\
\text { d.c.c } \\
\text { b.c.d } \\
\text { c.b.d } \\
\text { c.a.c }\end{array}$ \\
\hline 3 & $\begin{array}{l}\text { (c.b.c.d, } \\
\text { a.c.d.d.c, } \\
\text { a.b.d.d.a.b.d) }\end{array}$ & $\begin{array}{l}\text { b.c.d } \\
\text { c.b.c }\end{array}$ & $\begin{array}{l}\text { a.b.d } \\
b . a . d\end{array}$ & a.c.d & $\begin{array}{l}\text { a.c.d } \\
\text { b.c.d } \\
\text { c.b.d } \\
\text { c.a.c }\end{array}$ \\
\hline
\end{tabular}

Table 7

\begin{tabular}{|c|c|c|c|c|c|}
\hline & $\mathbf{A}$ & B & $\mathbf{C}$ & D & $\mathbf{E}$ \\
\hline & $\begin{array}{l}(a, b, c, d) \text { Tu- } \\
\text { ple }\end{array}$ & $\begin{array}{l}\text { Tuple's partial } \\
a \text { coronas }\end{array}$ & $\begin{array}{l}\text { Tuple's partial } \\
b \text { coronas }\end{array}$ & $\begin{array}{l}\text { Tuple's partial } \\
c \text { coronas }\end{array}$ & $\begin{array}{l}\text { Tuple's partial } \\
d \text { coronas }\end{array}$ \\
\hline $\mathbf{1}$ & $\begin{array}{l}\text { (c.b.c.d, } \\
\text { a.c.c.d.c, } \\
\text { a.b.c.b.d.a.b.d, } \\
\text { a.c.b.c.a.c.c.b) }\end{array}$ & $\begin{array}{l}\text { d.c.b } \\
\text { c.b.c } \\
\text { b.d.c } \\
\text { c.d.c }\end{array}$ & $\begin{array}{l}\text { a.c.d } \\
\text { a.c.c } \\
\text { c.c.d } \\
\text { c.a.c } \\
\text { c.d.a } \\
\text { c.d.c }\end{array}$ & $\begin{array}{l}\text { a.b.c } \\
\text { c.b.d } \\
\text { d.b.a } \\
\text { b.a.d } \\
\text { a.d.b } \\
\text { a.d.c } \\
\text { c.d.b }\end{array}$ & $\begin{array}{l}\text { b.c.a } \\
\text { c.b.c } \\
\text { c.a.c }\end{array}$ \\
\hline 2 & $\begin{array}{l}(c . b \cdot c \cdot d, \\
a . c . c . d . c, \\
a . b \cdot c \cdot b \cdot d \cdot a \cdot b \cdot d, \\
a . c . b \cdot c \cdot a \cdot c \cdot b \cdot c)\end{array}$ & $\begin{array}{l}\text { d.c.b } \\
\text { c.b.c } \\
\text { c.d.c }\end{array}$ & $\begin{array}{l}\text { a.c.d } \\
\text { a.c.c } \\
\text { c.c.d } \\
\text { c.a.c } \\
\text { c.d.c }\end{array}$ & $\begin{array}{l}\text { a.b.c } \\
\text { c.b.d } \\
\text { d.b.a } \\
\text { b.a.d } \\
\text { a.d.b }\end{array}$ & $\begin{array}{l}\text { b.c.a } \\
\text { c.b.c } \\
\text { c.a.c }\end{array}$ \\
\hline
\end{tabular}

Table 8

results in the patch illustrated in Figure 27. 


\begin{tabular}{|c|c|}
\hline 4-tuple & Proportions \\
\hline (d.d.d.d, c.d.c.d, b.d.d.b.d.d, a.d.a.d.c.b.c.d $)^{*}$ & $d=c+b$ \\
\hline (d.d.d.d, d.c.c.c, b.c.b.c.b.d.d, a.d.a.d.b.c.b.c.d)* & $d=c+b$ \\
\hline (c.d.c.d, d.d.d.d, a.d.d.a.d.d, a.c.d.b.d.b.d.c)* & $d=c+a$ \\
\hline (d.d.d.d, c.d.c.d, d.d.d.b.d,a.d.c.d.c.b.c.d $)^{*}$ & $d=c+b$ \\
\hline (c.d.c.d, d.d.d.d,a.d.d.d.d, a.c.d.c.d.b.d.c)* & $d=c+a$ \\
\hline (c.c.c.d, d.d.d.d, a.c.a.c.a.d.d, a.c.d.b.d.b.d.c)* & $d=c+a$ \\
\hline (d.d.c.c, c.d.c.d, a.c.a.d.b.d.d, a.c.d.a.c.b.c.d $)^{*}$ & $b=2 a ; d=c+a$ \\
\hline (d.b.c.d, a.d.d.d.c, a.b.d.a.b.d, a.c.b.d.b.d.b.a.d) & $d=2 a+2 b$ \\
\hline (d.b.c.d, a.d.d.d.c, a.b.d.a.b.d, a.c.b.d.b.d.a.b.d) & $d=2 a+2 b$ \\
\hline (d.b.c.d, a.d.c.a.d.c, a.b.d.d.d, a.c.d.c.d.c.b.a.d) & $b=3 a ; d=2 a+c$ \\
\hline (c.b.c.d,a.c.d.c.c, a.b.c.b.d.a.b.d,a.c.b.c.a.c.b.c) & $d=3 a$ \\
\hline (d.b.c.d, a.d.c.d.c, a.b.d.d.b.d, a.c.b.a.d.c.b.c.d) & $d=a+2 b$ \\
\hline (d.b.c.d, a.d.d.d.c, a.b.d.a.b.d, a.c.b.d.b.d.b.a.d) & $d=a+2 b$ \\
\hline (d.b.c.d,a.d.d.d.c, a.b.d.a.b.d,a.c.b.d.b.d.a.b.d) & $d=a+2 b$ \\
\hline (d.b.c.d, a.d.c.c.c, a.b.d.a.b.d,a.c.b.a.d.a.c.b.a.d) & $d=a+2 b$ \\
\hline (c.b.c.b, a.c.d.c.c, a.b.d.d.b.c.b, b.c.d.c.b.c.d.c) & $d=a+2 b$ \\
\hline (b.b.c.c, a.b.a.c.d.c, a.b.d.d.b.a.c, b.c.d.c.b.c.d.c) & $d=a+2 b$ \\
\hline (b.c.b.c, a.c.d.d.c, a.b.d.d.d.b, b.c.d.c.d.c.b.d) & $d=a+2 b$ \\
\hline (b.c.d.d, a.d.d.c.c, a.b.c.b.d.d,a.c.d.c.b.d.a.b.d) & $b=2 a ; d=a+2 b$ \\
\hline (c.b.c.b, a.c.d.d.c, a.b.d.d.d.b, b.c.d.c.d.b.c.d) & $d=a+2 b$ \\
\hline (d.b.c.d,a.d.c.a.d.c,a.b.d.b.a.d,a.c.a.d.a.b.c.b.a.d) & $d=a+2 b$ \\
\hline (d.b.c.d,a.d.d.c.c, a.b.c.b.d.c.d,a.c.c.b.d.a.b.d) & $d=2 a+b$ \\
\hline (d.b.c.d, a.d.c.a.d.c, a.b.d.c.d.d, a.d.c.a.d.c.c.b) & $b=2 a ; d=2 a+b$ \\
\hline (c.b.c.b,a.c.d.d.c, a.b.d.b.a.b.d.b, b.c.b.d.b.c.b.d) & $d=2 a+b$ \\
\hline (d.b.c.d,a.d.c.a.d.c, a.b.d.c.d.d, a.c.d.c.c.b.a.d) & $b=2 a ; d=2 a+b$ \\
\hline (d.b.c.d, a.d.c.a.d.c, a.b.d.d.d, a.c.d.c.d.c.b.a.d) & $d=2 a+b$ \\
\hline (d.b.c.d, a.d.d.c.c, a.b.c.d.b.c.d, a.c.c.b.d.a.b.d) & $b=2 a ; d=2 a+b$ \\
\hline (d.b.c.d, a.d.d.d.c, a.b.d.a.b.d, a.c.b.d.b.d.b.a.d) & $d=2 a+b$ \\
\hline (d.b.c.d,a.d.d.c.c, a.b.c.b.d.c.d, a.c.c.b.d.b.a.d) & $b=2 a ; d=2 a+b$ \\
\hline (c.b.c.d, a.c.c.a.c.c, a.b.c.b.a.d.d, a.c.d.c.a.c.d.c) & $d=2 a+b$ \\
\hline$($ d.b.c.d, a.d.d.d.c, a.b.d.a.b.d, a.c.b.d.b.d.b.a.d) & $d=2 a+b$ \\
\hline (c.b.c.d, a.c.d.d.c, a.b.d.b.a.d.d, a.c.d.b.c.b.d.c) & $d=2 a+b$ \\
\hline (c.b.c.c, a.c.d.d.c, a.b.d.b.a.c.a.c, b.c.b.d.b.c.b.d) & $d=2 a+b$ \\
\hline (c.b.c.d,a.c.d.d.c, a.b.d.a.b.d.d, a.c.d.c.b.d.b.c) & $d=2 a+b$ \\
\hline (c.b.c.d, a.c.d.d.c, a.b.d.a.b.d.d, a.c.d.b.c.d.b.c) & $d=2 a+b$ \\
\hline (c.b.c.d, a.c.d.d.c, a.b.d.d.d, a.c.d.c.d.c.b.d.c)** & $d=2 b+a$ \\
\hline (c.b.c.d., a.c.d.d.c., a.b.d.c.d.d., a.c.d.c.c.b.d.c)** & $d=2 a+b$ \\
\hline (d.b.c.d., a.d.c.a.d.c., a.b.d.b.a.d.d., a.c.d.a.b.c.b.a.d $)^{* *}$ & $d=2 a+b$ \\
\hline (c.d.c.d., c.c.d.d., a.d.b.c.d.d., a.c.d.c.c.d.b.c) ${ }^{* *}$ & $d=2 a+b$ \\
\hline (c.d.c.d., c.c.d.d., a.d.b.c.b.d.d., a.c.d.b.c.d.b.c)** & $d=2 a+b$ \\
\hline
\end{tabular}

Table 9: All 4-tuples generated when $a+b=c$. Note that all marked with $(*)$ were already found in Section 2.1. All marked with (**) cannot be extended to create a tiling of the plane. 


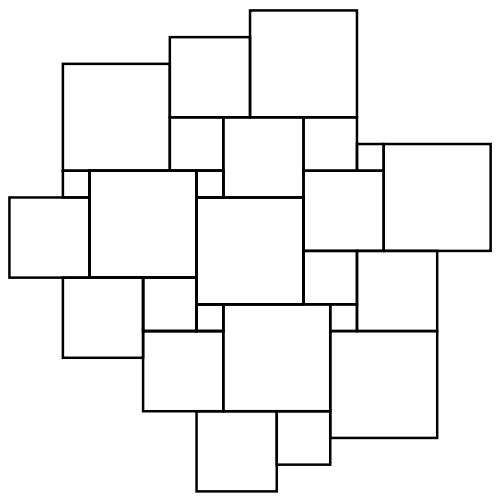

Figure 27: A second layer of the $d$ corona for (d.b.c.d, a.d.d.c.c, a.b.c.b.d.c.d, a.c.c.b.d.b.a.d).

By adding tiles in a similar manner, such that the coronas are reflections and rotations of those in Figure 26, as well as deleting tiles where necessary, one will easily find a patch which can tile the plane unilaterally and equitransitively using translations. Such a patch is illustrated in Figure 28.

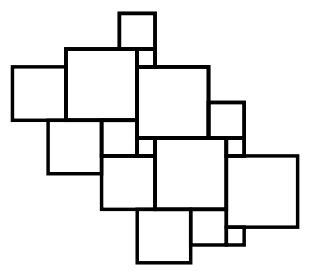

Figure 28: A patch which will tile the plane unilaterally and equitransitively by way of translations.

However, for some 4-tuples, it will soon become clear that no tiling is possible. For example, consider the 4-tuple (c.b.c.d, a.c.d.d.c, a.b.d.d.d, a.c.d.c.d.c.b.d.c), for which $c=a+b$ and $d=a+2 b$. If one attempts to expand on the $d$ corona in a similar manner as above - by completing incomplete coronas while adhering to the ordering prescribed by the 4-tuple - one will encounter the patch in Figure 29.

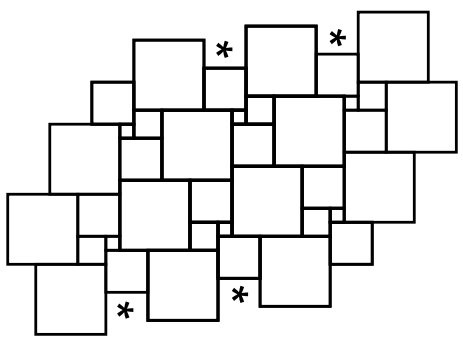

Figure 29: A patch which cannot be extended for 4-tuple (c.b.c.d, a.c.d.d.c, a.b.d.d.d, a.c.d.c.d.c.b.d.c), for which $c=a+b$ and $d=a+2 b$. 
The $(*)$ represent problem areas. To adhere to equitransitivity, one must place a $d$ tile in these spots. Obviously, this is impossible. For each of the five tilings marked with (**) in Table 9, a patch which could not be extended was inevitable.

\section{$5 a$ and $b$ are not adjacent}

In this case, there are six possible $a$ coronas. These are illustrated in Figure 30. By replacing the $a$ tiles with $b$ tiles in Figure 30, it is clear that there are also exactly six $b$ coronas when $a$ and $b$ are not adjacent. Lemma 5.1 eliminates one of these six subcases of $a$ coronas and one of these six subcases of $b$ coronas from consideration in UET4 tilings.

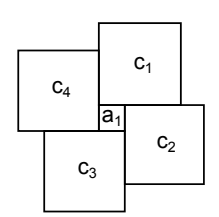

(a)

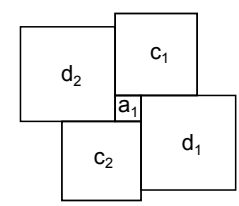

(d)

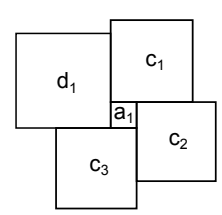

(b)

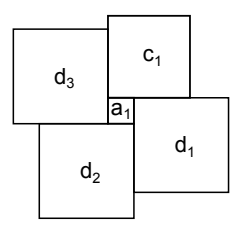

(e)

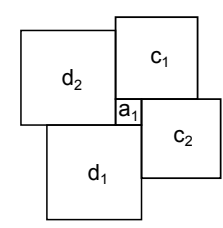

(c)

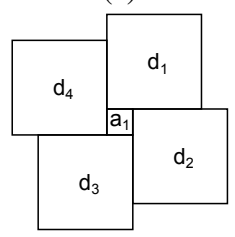

(f)

Figure 30: All possible $a$ coronas when $a$ and $b$ are not adjacent

Lemma 5.1. Let $\mathscr{T}$ be a UET4 tiling in which $a$ and $b$ are not adjacent. Then a coronas and $b$ coronas cannot contain only c tiles.

Proof. Suppose that the $a$ corona can contain only $c$ tiles as shown in Figure 31a below. At least one corner formed by two tiles $c$ must contain a non- $a$ tile; otherwise the resulting tiling would be UET2. Without loss of generality, suppose that this required corner is that marked by the asterisk in Figure 31a; it will be determined which tiles can be placed in the corner marked by the asterisk. Were a $b$ tile to be placed here, then this $b$ tile would overhang past the right edge of $c_{1}$. Since every $b$ tile is a vortex, the length of this overhang must be covered exactly by a tile (or tiles), and the only tile that can cover this length $b-a$ while maintaining the appropriate relative side lengths of $a, b, c$, and $d$ is an $a$ tile. However, this would contradict $a$ and $b$ not being adjacent. Therefore a $d$ tile must fill this space as shown in Figure 31b. Next, it is determined which tiles could be placed in the corner marked by the asterisk in FIgure 31b. Were an $a$ tile to be placed here, there would be a $d$ tile in its corona and the tiling would cease to be equitransitive by the assumption that $a$ 's corona contained only $c$ tiles. A $c$ tile cannot fill the asterisked corner by unilaterality, so the two cases shown in Figures 31c and 31d must be considered, starting with Figure 31c. 


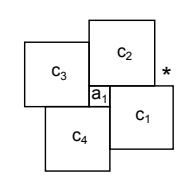

(a)

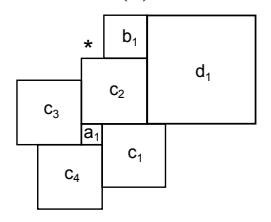

(d)

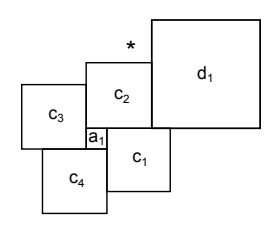

(b)

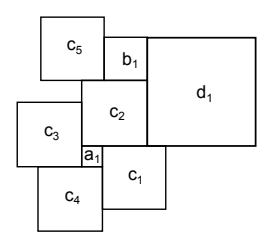

(e)

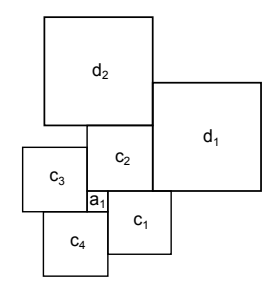

(c)

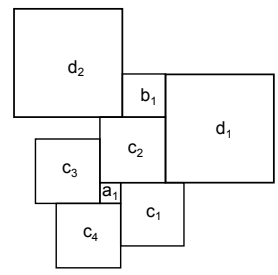

(f)

Figure 31: Progressively building all possible $c$ coronas

The vertical distance remaining along the left edge of tile $c_{2}$ in this figure is of length $a$; hence the only tile that could appropriately fill this remaining edge length is an $a$ tile. However, the corona of this new $a$ tile would contain a $d$ tile, contrary to hypothesis. Therefore the arrangement of tiles in Figure 31c does not give rise to a UET4 tiling.

Next consider case illustrated in Figure 31d. Note that, as pictured, the top edge of tile $b_{1}$ must line up with the top edge of $d_{1}$. Were this not the case, either $b_{1}$ would cease to be a vortex or would be forced to have an $a$ tile in its neighborhood. Thus, an $a$ tile cannot be placed in the corner marked by the asterisk because $a$ and $b$ cannot be adjacent; neither can a $b$ tile be placed there by the unilaterality condition. This leaves the two cases shown in Figure 31e and 31f. In both of these cases, the remaining vertical distance along the left edge of $c_{2}$ is of length $a$, so the only tile that could fill this space is an $a$ tile. However, the distance that the bottom edge of $c_{5}$ in Figure 31e and the bottom edge of $d_{2}$ in Figure $31 \mathrm{f}$ hang over the left edge of $c_{2}$ is, in both cases, strictly greater than the length $a$. Hence an $a$ tile placed along the remaining left edge of $c_{2}$ would not be a vortex. Therefore the arrangement of tiles found in Figure 31d does not give rise to any UET4 tilings.

A nearly identical argument shows that a $b$ tile cannot be surrounded by only $c$ tiles.

Because neither an $a$ tile nor a $b$ tile can have a corona containing only $c$ tiles, then each of their coronas must contain a $d$ tile. An immediate corollary to this is that each $d$ corona must contain at least one $a$ tile and at least one $b$ tile. Lemma 5.1 implies that there are five possible $a$ coronas and five possible $b$ coronas when $a$ and $b$ are not adjacent.

Lemma 5.1 illustrates the analysis of only one possible $a$ corona, but there are 5 more $a$ coronas to consider, 6 more $b$ coronas, and several possible $c$ and $d$ coronas to consider. Because each possible corona involves exhaustive examination, it would be impractical to present such an analysis in a short article. However, the following example illustrates the methodology used to decide if a given corona is viable. Consider the $a$ corona of Figure 30d. This corona is reprinted in Figure 32a. Proceed by constructing all possible $d$ coronas that arise from this arrangement by placing tiles along the edges of the tile $d_{2}$ in Figure 32a moving in a clockwise direction. There are three subcases here to consider: 
$d>a+c, d=a+c$, and $d<a+c$. In this example, only the subcase $d=a+c$ is demonstrated. To begin enumeration of all possible $d$ coronas that can arise from the arrangement in Figure 32a, first determine which tiles could be placed in the corner marked with an asterisk. An $a$ tile cannot be placed there because the vortex restriction on $a$ tiles would imply that $c=d$. A $c$ tile cannot be placed there by unilaterality. This gives us two options to consider: a tile $b_{1}$ can be placed there or a tile $d_{3}$ can be placed there. These two options are shown in Figures 32b and 32c. Note that for the case shown in Figure 32b, the vortex condition on $b$ tiles and the fact that $a$ tiles and $b$ tiles cannot be adjacent requires that $a+d=b+c$.

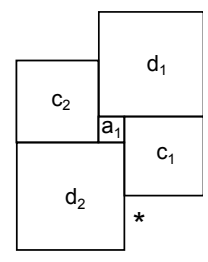

(a)

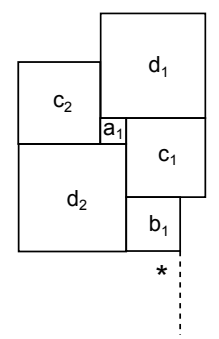

(b)

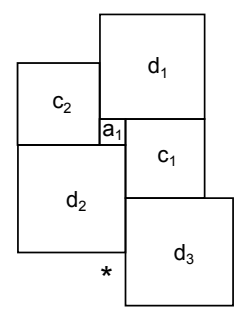

(c)

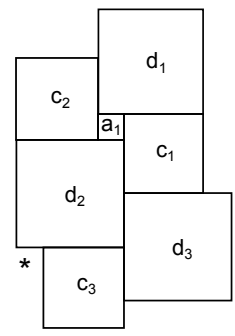

(d)

Figure 32

In a full analysis, both of the arrangements in $32 \mathrm{~b}$ and $32 \mathrm{c}$ above need to be considered, but for the purposes of this example, consider only how to fill in the asterisked corner in Figure 32c. A $d$ tile cannot be placed there by unilaterality; however, an $a$, a $b$, or a $c$ could be placed there under the appropriate conditions. Each of these options needs to be considered. Examine the arrangement, shown in Figure 32d, where a tile $c_{3}$ fills the asterisked corner. Again, it must be determined which tiles can be placed in the asterisked corner in Figure 32d. Were a tile $b$ placed there, the condition $d=a+c$ implies that the top edge of $b$ will have overhang past the left edge of $d_{2}$; then in order for $b$ 's vortex condition to be satisfied, a tile $a$ would have to be placed along the remaining top edge of $b$. This contradicts $a$ and $b$ not being adjacent. A $c$ tile cannot be placed in the asterisked corner by unilaterality. This leaves two options to consider. The case an $a$ tile in this position is seen in Figure 33a, and the case where a $d$ tile in this position is seen in Figure 34a.

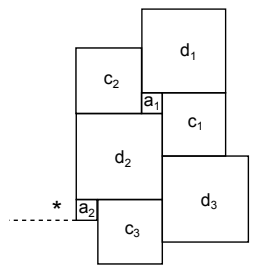

(a)

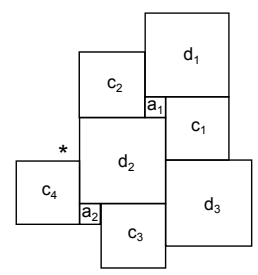

(b)

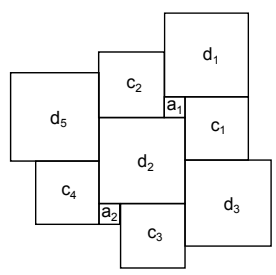

(c)

Figure 33

Consider first the arrangement shown in Figure 33a. By equitransitivity of $a$, a tile $c_{4}$ must be placed in the asterisked corner so that its corona will match that of $a_{1}$. This 
is shown in Figure 33b. The asterisked corner Figure $33 \mathrm{~b}$ can only be filled by a tile $d_{4}$, shown in Figure 33c; $a$ and $b$ are not allowed there by the vortex conditions, and $c$ is not allowed by unilaterality. Then Figure $33 \mathrm{c}$ shows a completed $d$ corona. However, since this $d$ corona contains no $b$ tile, this will not result in a UET4 tiling as a result of a corollary to Lemma 5.1 and is hence not a viable $d$ corona.

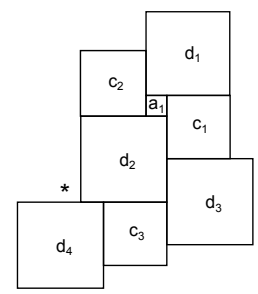

(a)

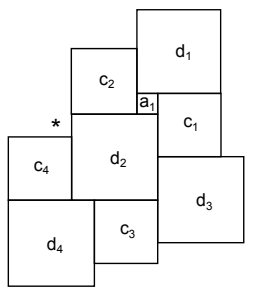

(b)

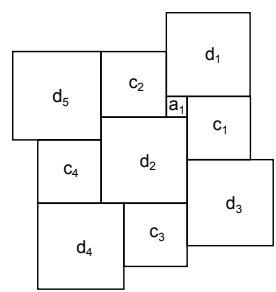

(c)

Figure 34

Next, considering the arrangement shown in Figure 34a, it must be determined which tiles can be placed in the corner marked by an asterisk. Vortex conditions prohibit an $a$ or $b$ from being placed there, and a $d$ tile is also not allowed by unilaterality. Then the only option is to place a tile $c_{4}$ in this position, shown in Figure 34b. The asterisked corner in this figure can only be filled by a tile $d_{5}$, as shown in Figure 34c; $a$ and $b$ are not possible by vortex conditions and $c$ is not possible by unilaterality. Figure $34 \mathrm{c}$ shows a complete $d$ corona. However, because this corona does not contain a $b$ tile, it is not compatible with a UET4 tiling.

The next step would be to consider the possibilities when an $a$ tile or a $b$ tile are placed in the position occupied by $c_{3}$ in Figure $32 \mathrm{~d}$. The method continues in this fashion, enumerating all possible tiles that can be placed in a location, moving around a $d$ tile, creating a branching list of all $d$ coronas and weeding out coronas that are known to be impossible under our constraints. This is done for the three subcases $c>a+c, d=a+c$, and $d<a+c$ for each of the five possible $a$ coronas in Figures 30b-30f, and it seen that $d=a+c$ is the only case that yields viable $d$ coronas. It should also be noted that in the case where an $a$ tile is surrounded by three $c$ tiles and one $d$ tile, as shown in Figure 30b, the same method used to build around a $c$ tile instead of a $d$ tile. It should also be noted that, at times, it is necessary to specify certain side lengths for $b$ and $c$ tiles in terms of side lengths of smaller tiles in order for certain arrangements to be viable. This allows for further flexibility in corona construction and ensures that all possible potentially viable coronas are found.

Summarizing, the criteria used throughout this method are as follows:

1. Equitransitivity of the tiling $\mathscr{T}$.

- When an $a$ tile is placed in the corona of a larger tile, it is possible to continue building around the larger tile using the knowledge that every $a$ neighborhood must be identical to that of the already established $a_{1}$ corona.

- It is possible that at times the only option is to place a tile within an $a$ tile's neighborhood that makes it incompatible with the original $a_{1}$ corona. In this case, the method can be ended on this branch, as it will not yield any viable $c$ or $d$ coronas. 
2. Unilaterality of the tiling $\mathscr{T}$.

3. Vortex conditions on $a$ and $b$ tiles.

4. The requirement that $a$ tiles and $b$ tiles are not adjacent.

5. Relative sizes of tile side lengths: $a<b<c<d$.

6. Each $d$ corona must contain at least one $a$ tile and at least one $b$ tile.

This method essentially begins with one of the five viable $a$ coronas shown in Figure 30 and then employs the corona construction algorithm outlined previously in Section 3, building around a tile in the $a$ corona (specifically, around a $d$ tile in Figures 30c, 30d, and $30 \mathrm{e}$ or around a $c$ tile in Figures 30a and 30b) until either a contradiction is reached for a particular branch or a full corona is reached. Once the construction process has been completed building around the chosen tile in the corona of the original $a$ tile, one is left with an exhaustive list of all $d$ coronas (or $c$ coronas, depending on the $a$ corona from which construction began) that are compatible with the original $a$ corona. Then, using equitransitivity, the coronas of all new $a$ and $d$ tiles (or $c$ tiles, again depending on the case) can be completed, expanding the patch until one can either establish that no UET4 tiling can result (due to failure of equitransitivity, overlapping or gaps between tiles, contradiction of vortex conditions, etc.) or until full $b$ and $c$ coronas (or $b$ and $d$ coronas) are found. Note that multiple tilings may result from the same set of $a$ and $d$ coronas (or $a$ and $c$ coronas), as there may be multiple ways to tile the original patch using these coronas. Table 10 lists the eleven UET4 tilings found using this method and the necessary side length proprtions required for the tiling to be generated.

\begin{tabular}{|c|c|}
\hline ( $a$ corona, $b$ corona, $c$ corona, $d$ corona) & Side Relations \\
\hline (d.d.d.d, c.d.c.d,b.d.d.b.d.d, a.d.a.d.c.b.c.d) & $c=a+b ; d=c+b$ \\
\hline (d.d.d.d, d.c.c.c, b.c.b.c.b.d.d, a.d.a.d.c.b.c.d) & $c=a+b ; d=c+b$ \\
\hline (c.d.c.d, d.d.d.d, a.d.d.a.d.d, a.c.d.b.d.b.d.c) & $c=a+b ; d=c+a$ \\
\hline (d.d.c.c, c.d.c.d, b.d.d.a.c.a.d,a.d.a.c.d.c.b.c) & $b=2 a ; d=c+a$ \\
\hline (d.d.d.d, c.d.c.d, d.d.d.b.d,a.d.c.d.c.b.c.d) & $c=a+b ; d=c+b$ \\
\hline (c.d.c.d, d.d.d.d, a.d.d.d.d, a.c.d.c.d.b.d.c) & $c=a+b ; d=c+a$ \\
\hline (c.d.c.d, c.d.c.d, a.d.b.d.d.d,b.c.a.c.d.c.d.c) & $b=2 a ; d=c+a$ \\
\hline (c.c.c.d, d.d.d.d, a.c.a.c.a.d.d, a.c.d.b.d.b.d.c) & $c=a+b ; d=c+a$ \\
\hline (d.d.c.c, c.d.c.d, a.c.a.d.b.d.d,a.c.d.a.c.b.c.d) & $b=2 a ; c=a+b ; d=c+a$ \\
\hline (c.d.c.d,c.c.d.d, a.d.b.c.b.d.d,a.c.b.d.c.b.d.c) & $b=2 a ; c=2 b ; d=c+a$ \\
\hline (c.d.c.d, c.c.d.d, b.c.b.d.d.a.d,a.c.d.c.b.d.b.c) & $b=2 a ; d=c+a$ \\
\hline
\end{tabular}

Table 10

Illustrations of the eleven tilings when $a$ and $b$ are not adjacent can be seen in the final section. This concludes the case where $a$ tiles and $b$ tiles are not allowed to be adjacent. 


\section{The 39 UET4 Tilings}

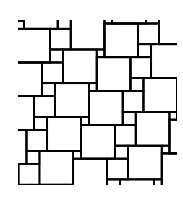

(d.d.d.d, c.d.c.d, b.d.d.b.d.d, a.d.a.d.c.b.c.d) $c=a+b$ $d=c+b$

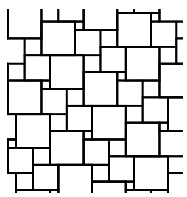

(c.d.c.d,

c.c.d.d, a.d.b.c.b.d.d, a.c.d.c.b.d.b.c)

$b=2 a$

$d=c+a$

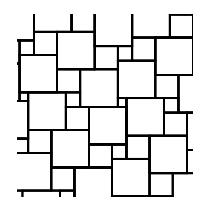

(d.d.d.d, d.c.d.c , b.d.d.d.d, a.d.c.d.c.b.c.d) $c=a+b$ $d=c+b$

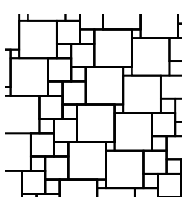

(d.d.d.d, c.c.c.d, b.c.b.d.d.b.c, a.d.c.b.c.d.a.d) $c=a+b$ $d=b+c$

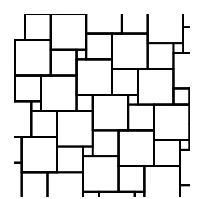

(c.d.c.d, d.d.d.d a.d.d.d.d, a.c.d.c.d.b.d.c) $c=a+b$ $d=c+a$

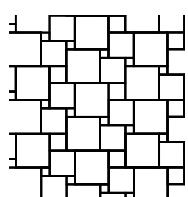

(c.c.d.d, c.d.c.d, a.c.a.d.b.d.d a.d.a.c.d.c.b.c) $b=2 a$ $d=c+a$

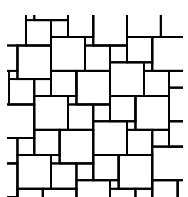

(c.d.c.d, c.d.c.d, a.d.b.d.d.d, a.c.d.c.d.c.b.c) $b=2 a$ $d=c+a$

Below are the 28 tilings when a and $\mathrm{b}$ are adjacent. For all, $c=a+b . d=a+b$ does not generate any tilings.

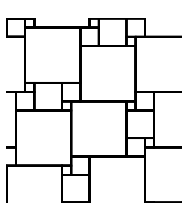

(d.b.c.d, a.d.d.d.c, a.b.d.a.b.d, a.c.b.d.b.d.a.b.d) $d=2 a+2 b$

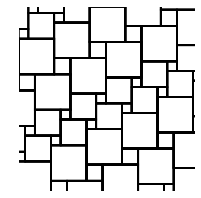

\section{(c.c.c.d}

d.d.d.d,

a.c.a.c.a.d.d, a.c.d.b.d.b.d.c)

$c=a+b$

$d=c+a$

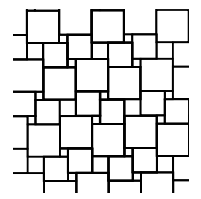

(d.d.c.c,

c.d.c.d,

a.c.a.d.b.d.d, a.c.d.a.c.b.c.d)

$b=2 a$

$c=a+b$

$d=c+a$

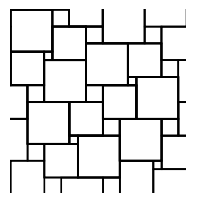

(c.d.c.d,

c.c.d.d,

a.d.b.c.b.d.d,

a.c.b.d.c.b.d.c)

$b=2 a$

$c=2 b$

$d=c+a$

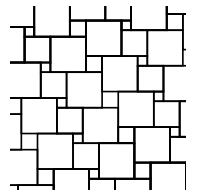

(c.d.c.d,

d.d.d.d, a.d.d.a.d.d, a.c.d.b.d.b.d.c)

$c=a+b$

$d=c+a$

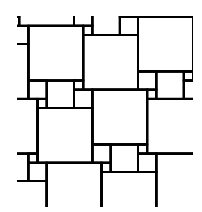

(d.b.c.d, a.d.d.d.c, a.b.d.a.b.d, a.c.b.d.b.d.b.a.d) $d=2 a+2 b$

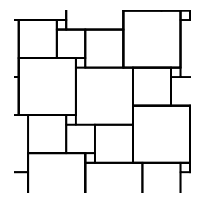

(d.b.c.d, a.d.c.a.d.c, a.b.d.d.d, a.c.d.c.d.c.b.a.d) $b=3 a$ $d=2 a+c$

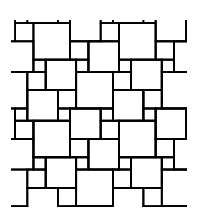

(c.b.c.d, a.c.d.c.c, a.b.c.b.d.a.b.d, a.c.b.c.a.c.b.c) $d=3 a$

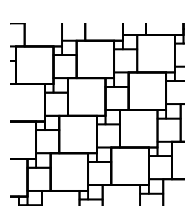

(d.b.c.d

a.d.c.d.c,

a.b.d.d.b.d,

a.c.b.a.d.c.b.c.d)

$d=a+2 b$

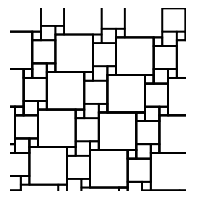

(d.b.c.d, a.d.c.a.d.c, a.b.d.a.b.d, a.c.b.a.d.a.c.b. a.d) $d=a+2 b$ 


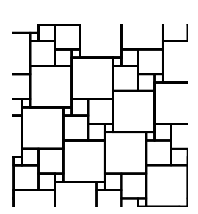

(d.b.c.d,

a.d.d.c.c, a.b.c.b.d.d, a.d.a.c.d.c.b.d.b) $d=a+2 b$

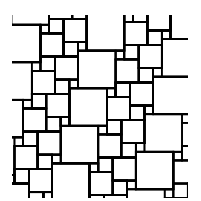

(d.b.c.d,

a.d.c.c.c, a.b.c.b.c.b.d, a.c.b.a.d.a.c.b. a.d) $d=a+2 b$

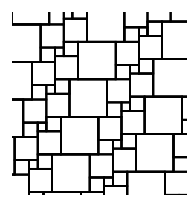

(c.b.c.b a.c.d.c.c, a.b.d.d.b.c.b b.c.d.c.b.c.d.c) $d=a+2 b$

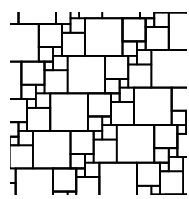

(b.b.c.c, a.b.a.c.d.c, a.b.d.d.b.a.c b.c.d.c.b.c.d.c) $d=a+2 b$

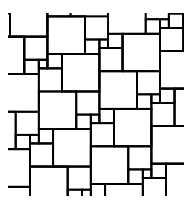

(b.c.b.c, a.c.d.d.c, a.b.d.d.d.b b.c.d.c.d.c.b.d) $d=a+2 b$

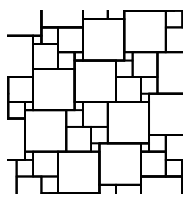

(b.c.d.d, a.d.d.c.c, a.b.c.b.d.d, a.c.d.c.b.d.a. b.d)

$b=2 a$

$d=a+2 b$

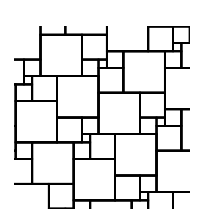

(c.b.c.b, a.c.d.d.c a.b.d.d.d.b, b.c.d.c.d.b.c.d) $d=a+2 b$

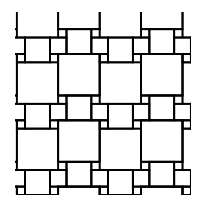

(d.b.c.d, a.d.c.a.d.c, a.b.d.b.a.d, a.c.a.d.a.b.c.b $a . d)$
$d=a+2 b$

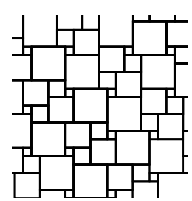

(d.b.c.d, a.d.d.c.c, a.b.c.b.d.c.d, a.c.c.b.d.a.b.d) $d=2 a$ $d=2 a+b$

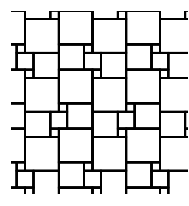

(d.b.c.d, a.d.c.a.d.c a.b.d.c.d.d, a.d.c.a.d.c.c.b) $b=2 a$ $d=2 a+b$

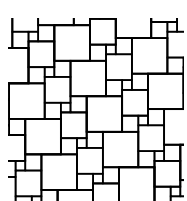

(c.b.c.b, a.c.d.d.c, a.b.d.b.a.b.d.b, b.c.b.d.b.c.b.d) $d=2 a+b$

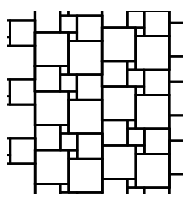

(d.b.c.d, a.d.c.a.d.c, a.b.d.c.d.d, a.c.d.c.c.b.a.d) $b=2 a$ $d=2 a+b$

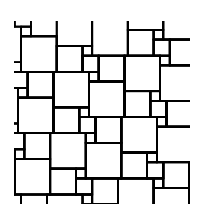

(d.b.c.d,

a.d.c.a.d.c, a.b.d.d.d, a.c.d.c.d.c.b.a.d) $d=2 a+b$

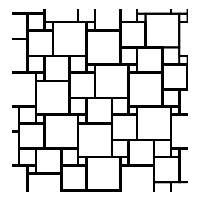

(d.b.c.d, a.d.d.c.c, a.b.c.d.b.c.d, a.c.c.b.d.a.b.d) $b=2 a$ $d=2 a+b$

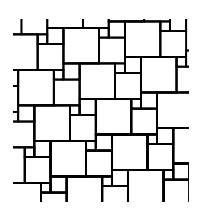

(b.c.d.c

a.c.d.d.c, a.b.d.b.a.d.d a.c.d.b.c.b.d.c) $d=2 a+b$

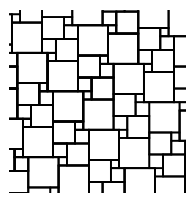

(d.b.c.d, a.d.d.c.c, a.b.c.b.d.c.d, a.c.c.b.d.b.a.d) $b=2 a$ $d=2 a+b$

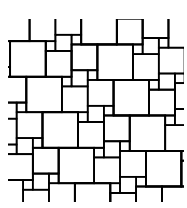

(c.b.c.d, a.c.c.a.c.c, a.b.c.b.a.d.d, a.c.d.c.a.c.d.c) $d=2 a+b$

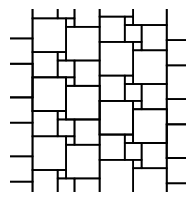

(b.c.d.d, a.d.c.a.d.c, a.b.d.d.c.d, a.c.c.d.c.b.a.d) $d=2 a$ $d=2 a+b$

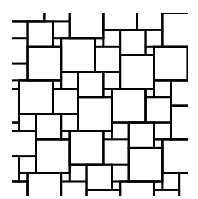

(b.c.d.d, a.c.c.d.d, a.b.c.d.b.c.d, a.c.c.b.d.a.b.d) $d=2 a+b$

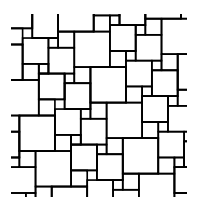

(c.b.c.c,

a.c.d.d.c,

a.b.d.b.a.c.a.c, b.c.b.d.b.c.b.d) $d=2 a+b$

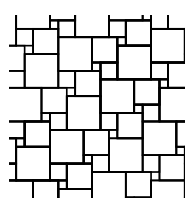

(c.b.c.d,

a.c.d.d.c,

a.b.d.a.b.d.d,

a.c.d.b.c.d.b.c)

$d=2 a+b$

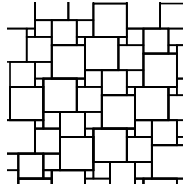

(c.b.c.d,

a.c.c.d.d, a.b.c.d.b.c.d, a.c.c.b.d.b.a.d) $b=2 a$ $d=2 a+b$ 


\section{References}

[1] G. Grünbaum and G. C. Shephard, Tilings and Patterns, Freeman, New York, 1987.

[2] H. Martini, E. Makai and V. Soltan, Unilateral tilings of the plane with squares of three sizes, Beiträge Algebra Geom. 39 (1998), 481-495.

[3] D. Schattschneider, Unilateral and Equitransitive Tilings by Squares, Discrete Comput. Geom. 24 (2000), 519-526. 